\title{
MERGED SIMULATION PROCEDURE FOR W-BAND IMAGING SYSTEMS
}

\author{
J. Gutiérrez, J. P. Pascual, A. Tazón \\ Department of Communications Engineering, University of Cantabria, 39005, Santander, SPAIN \\ (e-mail: pascualp@unican.es)
}

\author{
Shortened Title: \\ Merged Imaging System Simulation \\ Key words: \\ Detectors, Receivers, Radiometers, W band, Antenna, 3D EM, Non-Linear Circuits
}

\begin{abstract}
This paper presents and discusses a unifying simulation procedure aimed at providing more realistic performance prediction of basic imaging systems, combining 3D EM (3 Dimensions Electro-Magnetic) simulations and non-linear receiver circuit simulation. New methods are proposed to emulate the radiated emissivity profile of a target body and to use harmonic balance simulation to emulate non linear circuit/system response to a broadband noisy stimulus. The procedure focuses mainly on passive imaging, but active imaging including illuminated passive imaging is dealt with and some elemental experiments are presented and discussed for comparisons. The method enables the extrapolation of the frequency response of a single pixel detector to an array with more elements suitable for providing a complete set of pixels and can help in evaluating the most suitable bandwidth according to the operation mode (passive/active), frequency range of operation, desired resolution and the pixel number-image resolution trade-off.
\end{abstract}

\section{INTRODUCTION}

Imaging systems are formed by two parts: a radiating part conceived to receive a microwave signal from an emitting source-target (or reflected by a target after being previously sent in active systems), and a receiver part to process the received signal, extracting the information from the target. The radiating part is designed bearing in mind the size and distance to the target, the required resolution, number of pixels/number of receivers, capture procedure (simultaneous measurement of all pixels -focal plane arrayor mechanical/electrical sweep with fewer pixels/receivers -pixel scanning-), etc.

The receivers' circuit simulation is usually done with nonlinear simulation tools using Harmonic Balance (HB) or transient envelope algorithms, depending on the type of receiver and the signal processed (single tones, narrow or broadband pass-band signals or broadband noise). For a basic evaluation of the receiver with single tones the former is preferred. In the presence of modulated bandpass signals, the latter could be advisable. The complexity of the receiver scheme is also quite relevant for the choice of the design tools: a receiver architecture passing through several intermediate frequencies (IF) is more demanding, in terms of harmonic content and filtering than a total power receiver topology with direct detection, in which noise performance simulation could be quite relevant. Switching (like Dicke's radiometer) may provide an alternative to a total power radiometer to suppress some low frequency noise at the cost of lower sensitivity, and simulation correct procedure would require transient envelope simulation.

Imaging techniques [1],[2] cover a wide variety of applications including; radiometry to obtain the cosmic microwave background or remote sensing to plot soil moisture content maps of earth, medium and short range applications to detect concealed objects, small fractures in concrete structures or anomalies in biological tissues. Passive or active imagers are used, the former providing images in the form of temperature maps and the latter as reflectivity maps of the scene. Discussion of passive and active systems is presented in a practical approach in [3]. Within passive systems techniques have been proposed to enhance the capability of contrast [4] and to evaluate and decrease losses in the quasi-optical part of the imaging system [5].

Different scanning procedures have been studied pursuing optimization of the number of pixels and the hardware requirements [6]. There is also a huge amount of work studying the effect of frequencydependent antenna radiation patterns on the capability to provide an accurate image of a scene depending 
on far field or near field conditions in wide frequency bands or Continuous Wave (CW) systems. For example the concept of Millimeter-Wave Microscopes allows the use of a probe to overcome wavelength resolution limits [7]. Another alternative to obtain more information from a target at the cost of higher complexity is Frequency Modulated Continuous Wave (FMCW) radar, which has been successfully used in imaging systems [8]. Practical implementations of imaging systems have promoted a large number of proposals of patents, but summarizing them all is beyond the scope of this paper.

On the other hand, radiometer receiver simulation has been the object of several papers with emphasis on detection modeling in the frequency or time domain [9],[10]. For power budget purposes, receiver response is usually supposed to be flat in the band of interest. In [11] the effect of the road to and from a radar target is modelled using a built-in model which allows the integration with the rest of the circuitsystem simulation. Nevertheless limited attention has been paid to the interaction between receiver frequency response and the frequency profile of temperature or reflectivity/emissivity scene maps.

In this work a merged procedure to connect radiation profile and receiver response is proposed: first a reflectivity/emissivity or temperature pattern of an object, small enough to take advantage of W-band resolution, is assumed to be processed by an antenna providing a distribution of Electric Filed (E field) values in an X-Y front plane transformed by the multi-pixel-antenna-system into a two-dimensional (2-D) set of $\mathrm{E}$ field magnitudes. Those $\mathrm{E}$ magnitudes are converted into reflectivity/emissivity values and normalized to available power values, applied to the receiver chain (according to temperature and bandwidth estimations) to produce a 2-D map of detected output voltage which takes into account the frequency response of the receiver. This process is repeated sweeping the frequency value in the bandwidth of interest and, then, averaging the response to take into account both the broadband response of the system and the radiation pattern differences with frequency.

The method is conceived mainly for passive imaging as it requires broadband operation, but active imaging including both broadband noisy illuminated passive imaging and narrow band or fixed frequency will also be discussed for comparison.

The procedure may help in establishing the optimum bandwidth for maximizing contrast of the target and the optimum number of pixels to maintain acceptable resolution.

Initially considered scenarios for EM simulations are described distinguishing active and passive imaging. Then the circuit and system simulation procedures are described as well as the interface with EM simulations. Simple active and passive measurement setups are presented and compared with simulations.

The paper is intended to provide a flow diagram for the integration of circuit/simulation and EM design tools, which we consider of main relevance in the actual technology context where, for example, integration of circuits and antennas is becoming critical for system performance. In no case the goal of this work is to present a particular state-of-the-art performance system. Finally some conclusions are drawn.

\section{3D EM SIMULATION PROCEDURE}

Collection of radiation, reflected, emitted or both simultaneously by the target of an imaging system, is a very complex task to predict accurately, requiring the definition of some system and environment parameters (operation in near field or far field, use of lens, quasi-optics design, single antenna with mechanical/electrical sweeping, multiple antenna or a combination, etc.) and powerful 3D EM simulation tools, particularly for large scenarios. These Computed Aided Design (CAD) tools may provide field distributions or scattering parameter maps, which must be later exchanged with circuit-system CAD tools providing a global system output.

Two different simulation scenarios must be distinguished:

\section{A. Active Setup Scene}

It is possible to illuminate the sample with a front wave at a certain frequency and then to monitor the field in different positions, obtaining a total field map of the scene. For a more realistic simulation, easier to compare with measurements, antenna effect can be included: a horn antenna is positioned in front of a sample target with a hidden L-shape behind a dielectric layer. The antenna can be fed with a CW tone or with a broadband noisy signal. The last case would be quite similar to passive imaging, which will be discussed later. Using a CW tone, a port can be defined at the input of the WR-10 waveguide antenna feed and an $\mathrm{S}_{11}$ parameter can be obtained through the whole $\mathrm{W}$ band for a set of relative positions of the antenna with respect to the sample (see Figure 1), providing a reflectivity mapping image of the scene. 


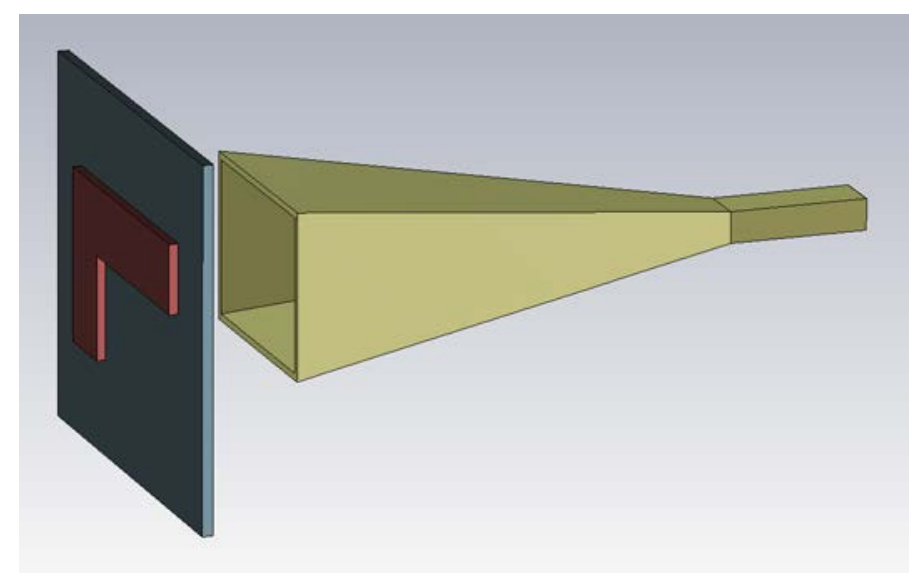

Figure 1. Sample with a hidden shape in front of W-band horn antenna

\section{B. Passive Setup Scene}

Conventional EM simulators do not provide the natural emission of a blackbody solid. Usually, a stimulus signal has to be defined in a frequency range. To emulate the emissivity profile of a sample target, a wave front linearly polarized is projected on the sample (see Figure 2). As the unity amplitude field is uniform in the incoming wave front, it is assumed that the field distribution at a given distance (the distance where the receiving antenna would be positioned) will contain the forward and backward components, being partially proportional to the reflectivity of the sample (see equations 1-8). In spite of relying on the capacity of the $\mathrm{W}$-band signals to penetrate objects, we will start the deduction assuming some opacity. If transmissivity is neglected (i.e.: opaque dielectric materials), it could be supposed that emissivity (equal to absorptivity by Kirchhoff's law) will be proportional to one minus reflectivity, providing a 2D emissivity profile which then could be normalized to a power level, according to the effective noise bandwidth of the receiver and the physical temperature of the sample, and then using that power as input for a receiver simulation. Polarization rotation and anisotropies will not be considered for simplicity.

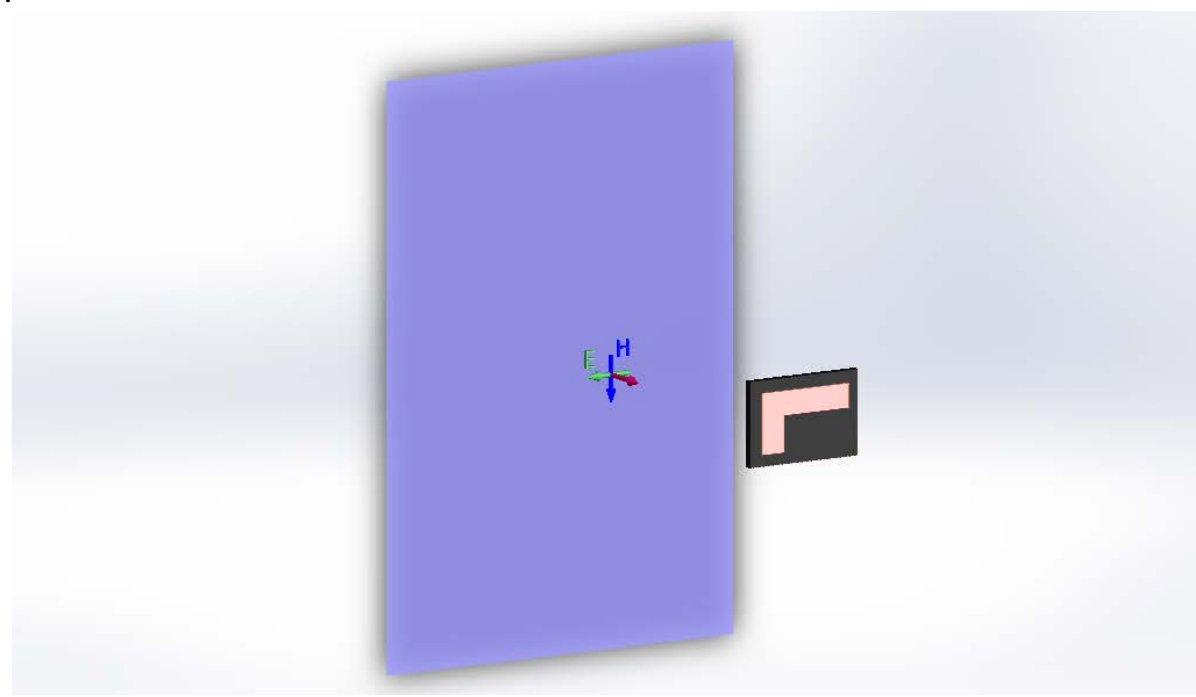

Figure 2. Wave front and sample to obtain an emissivity profile

The total E field at a fixed point includes incident and reflected field:

$$
\begin{gathered}
E_{t}=E_{i}+E_{r} \\
\frac{E_{t}}{E_{i}}=1+\Gamma
\end{gathered}
$$

Where we define a reflectivity factor: 


$$
\Gamma=\frac{E_{r}}{E_{i}}
$$

Assuming power conservation: reflectivity $\Gamma$, transmissivity $\mathrm{T}$, and absorptivity (equal to emissivity $\xi$ by Kirchhoff's law) are related by:

$$
\Gamma^{2}+T^{2}+\xi^{2}=1
$$

If the sample object is approached as opaque:

$$
T \approx 0
$$

Emissivity could be obtained as:

$$
\xi^{2}=1-\Gamma^{2}
$$

Radiometric temperature will be the product of emissivity and the physical temperature:

$$
T_{R}=\xi^{2} T_{\text {physical }}
$$

And the corresponding power, assuming an effective noise bandwidth BW $\mathrm{eff}_{\text {, }}$ and $\mathrm{K}$ being Boltzmann's constant:

$$
\text { Power }_{\text {emissivity }}=K T_{R} B W_{\text {eff }}=K T_{\text {physical }}\left(1-\Gamma^{2}\right) B W_{\text {eff }}
$$

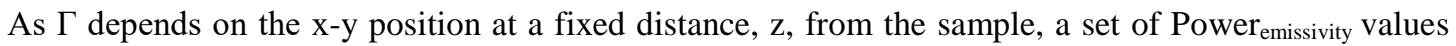
for every position could be obtained. Roughly, without considerations about field polarization or antenna radiation pattern, it could be supposed that an antenna placed in that position would collect that power, which would be added to the system noise and the whole could be detected and converted into a dc value for each position. A flow diagram of the procedure is presented in Figure 3. Receiver simulation is further discussed. Eventually, in a more accurate approach, the antenna radiation pattern could be considered, even with phase data available, the near field pattern could be deconvoluted taking into account scene geometry for a holographic reconstruction considering all the contributions to the field in a position at a certain distance from an aperture (such as the horn antenna) and avoiding the need of lens [12]. Even algorithms have been proposed to reconstruct holographic images from scalar data [13]. 


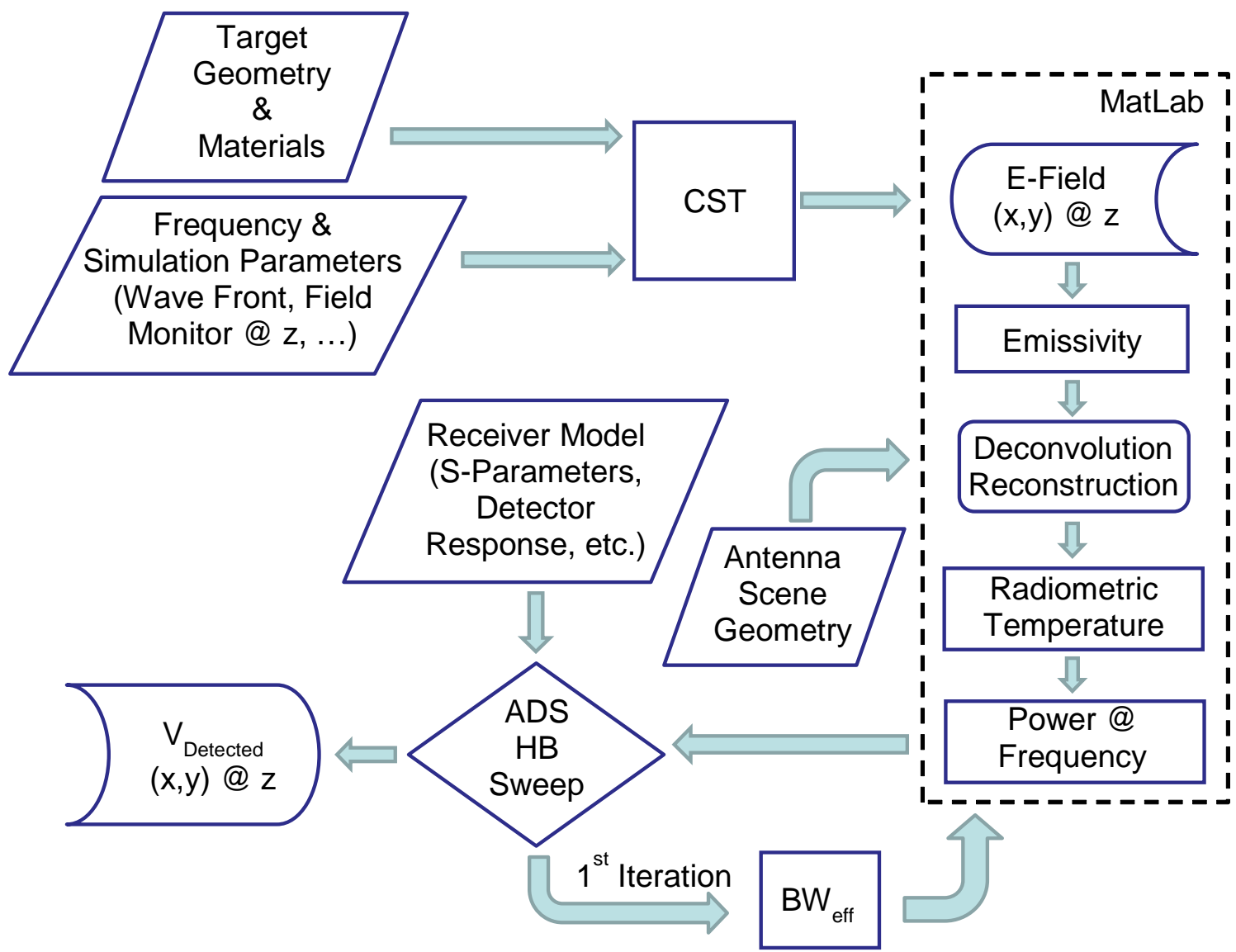

Figure 3. Flow diagram of the procedure to simulate passive imaging including frecuency response of the receiver combining EM (CST: Computer Simulation Technology), circuit-system (ADS: Advanced Design System) and mathematical simulation tools (MatLab)

\section{SIMULATION PROCEDURE AND FIGURES OF MERIT OF THE RECEIVER IN PASSIVE CONFIGURATION}

In a total power radiometer configuration the Effective Noise Bandwidth of a radiometer chain with gain $\mathrm{G}(\mathrm{f})$ is given by:

$$
B W_{\text {eff }}=\frac{\left[\int G(f) d f\right]^{2}}{\int G^{2}(f) d f}
$$

Where G(f) represents the Radio Frequency (RF) chain gain as a frequency function. Nevertheless, as the RF chain is usually ended with a detector which converts all the incoming RF power into a dc output level plus a low-pass filtered signal, depending on system dynamics, G(f) should take account of the frequency response of the detector.

Therefore the previous equation should be re-written in terms of detected output voltage, dependent on the input frequency. This requires evaluating the output dc voltage of the detector when a single tone at a frequency $f=f_{i}$ is applied, which implies in a first approach neglecting any intermodulation or spectral regrowth. $f_{i}$ is swept across the entire band of interest. This sweep is discrete with $\mathrm{N}$ points and a

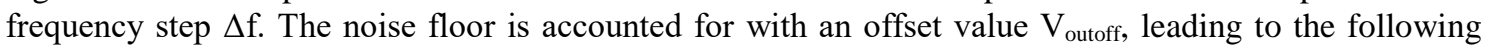
expression:

$$
B W_{\text {eff }}=\Delta f \cdot\left(\frac{N}{N+1}\right) \cdot \frac{\left(\sum_{i=1}^{N}\left(V_{\text {out }}\left(f_{i}\right)-V_{\text {outoff }}\right)\right)^{2}}{\sum_{i=1}^{N}\left(V_{\text {out }}\left(f_{i}\right)-V_{\text {outoff }}\right)^{2}}
$$


Actual operation of the radiometer in a pure passive or an illuminated passive setup would occur with a broadband noise input signal, whose power depends on the noise temperature of the input load. For characterization purposes, measurements based on sweeping frequency tones can be used [14]. To emulate this mode with Harmonic Balance (HB) simulations in the frequency domain, the performance of a frequency sweep of an "equivalent" tone is proposed. The effective noise bandwidth BW $\mathrm{eff}_{\text {is }}$ required to compute the equivalent tone power, therefore a previous sweep in harmonic balance is done with an approximate power to estimate the effective noise bandwidth under these conditions, and then, the resulting $\mathrm{BW}_{\text {eff, }}$ together with the values of equivalent noise temperature $\mathrm{T}_{\mathrm{e}}$ of the system and temperature of the intake charge $T_{R}$, are used to calculate the power that the equivalent-in-power tone to broadband noise should have, as shown in expression:

$$
P_{\text {Tone }}=K \cdot\left(T_{R}+T_{e}\right) \cdot B W_{\text {eff }}
$$

For these simulations, amplifiers could be modeled by their circuital models, if available, or could be previously characterized individually and represented by their corresponding measured S-parameters and noise figure, if compression is neglected, or by a system level amplifier block including S-parameters, noise figure, IP3 or $\mathrm{P}_{1 \mathrm{~dB}}$. Detector modeling in the simulations is more complex [10] as the nonlinear behavior is essential in this case, but with different response depending on the input frequency. In agreement with the simulation procedure a "Frequency Defined Device" (FDD) [15] is proposed after characterizing detector response to a set of single tones covering the band of interest. A two-port FDD enables voltages or currents at the output at a specific frequency (ie: dc) to be related to voltages or currents at the input at another frequency (ie: RF input tone) therefore inherently including the RF detector matching profile.

In the HB simulation, the input signal is swept in the band, concentrating all the power at each frequency step: a single tone with a power level equivalent to the broadband noise is frequency-swept in band to have approximately the same compression as in actual operation. An in deep discussion about compression under single tones or under broadband signals is out of our scope but it is dealt with in [16]. Therefore the dc output voltage is estimated as an average of the dc output values of the frequency sweeping in the operation band:

$$
V_{\text {outDC }}=\overline{V_{\text {out }}\left(f_{i}\right)} \quad \mathrm{i}: 1 \ldots \mathrm{N}
$$

\section{MEASUREMENT SETUPS}

A W-band horn antenna has been used as the main radiating element. Half beam width angle is estimated to be about $15^{\circ}$. The diameter of the horn is in the range of $17 \mathrm{~mm}$, Therefore, far field distance is about $175 \mathrm{~mm}$ at $94 \mathrm{GHz}$. The horn antenna is placed in a fixed position and a sample is placed in an xy holder to emulate mechanical scanning of a still sample by a moving antenna. The sample consists of a dielectric substrate $\varepsilon_{\mathrm{r}}=3.48,1.52 \mathrm{~mm}$ thick, with an L-shaped metal layer (17 $\mu \mathrm{m}$ thickness) in one case, or a glued $0.64 \mathrm{~mm}$ thick L-shaped piece with a different dielectric $\left(\varepsilon_{\mathrm{r}}=10.2\right)$ in the other case. In both cases, L-shaped piece size is $20 \mathrm{~mm} \times 12.5 \mathrm{~mm}$ with $5 \mathrm{~mm}$ width. The choice of this sample enabled better control of simulation parameters compared, for example, to a piece of cloth. In Figure 4, a photo of the L-shaped metal layer version is shown. 


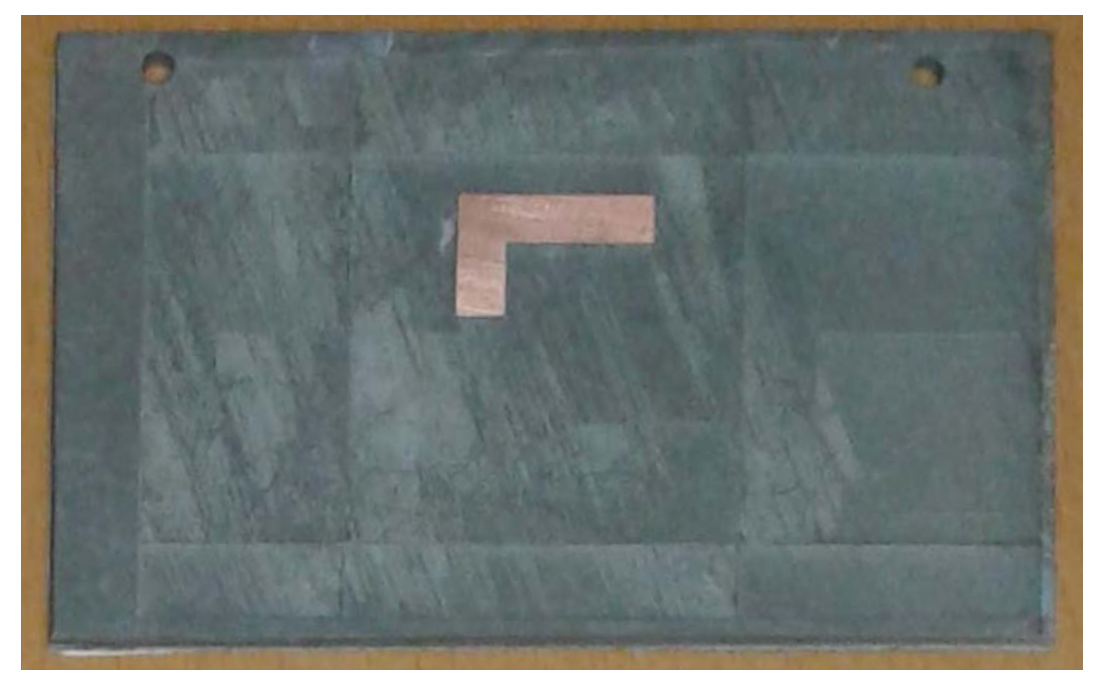

Figure 4. Photograph of the back of the sample: hidden L-shaped metal layer behind a dielectric layer

Taking into account the resolution required (order of magnitude of $\mathrm{mm}$ ) and the half beam width for a $\mathrm{W}$ horn, distance from the antenna to the sample is established in the near field zone, in the range of 7 $\mathrm{mm}$, a similar range to other experiments [17]. Unlike in other experiments like [5], no additional lenses are used in this close range configuration.

\section{A. Passive Setup}

A total power radiometer configuration is built using W-band Monolithic Microwave Integrated Circuits (MMIC) Low Noise Amplifiers (LNAs) mounted inside W-band WR-10 waveguide-access cases, cascading at least 2 units to provide enough gain and power to excite the detector (see Figure 5). Optionally, a band-pass filter (BPF) used in [18][19] can be added for band conformation, but at the risk of reducing, not only input noise, but also input signal power. In fact, for an indoor scene, maximum sensitivity would be required, a feature that additional RF gain would not guarantee, as was later verified, with the trade-off of chain saturation narrowing the dynamic range. The LNA's chain was constituted by two cascaded MMICs manufactured by Hughes (HRL). The nominal noise figure was expected to be about $3.5 \mathrm{~dB}$ but final assemblies including transition and bonding losses showed higher figures in the range of 5-7 dB (see Figure 6 and Figure 7). Figure 8 shows the LNA's cascade response including an isolator (labelled ISO) in between to reduce ripple due to mismatching. In TABLE I, the effective noise bandwidth $\left(\mathrm{BW}_{\text {eff }}\right)$ of the RF chain elements is summarized, comparing with an added band-pass filter, finding a decrease in $\mathrm{BW}_{\text {eff }}$ as expected, but also showing that the equivalent input noise level is in both cases higher than the input load level at room temperature.

For the detector, a waveguide-mounted commercial model fabricated by Hughes was used (Figure 9 shows sensitivity in $\mathrm{W}$ band). Individual characterization of each element was required prior to the global simulations. 


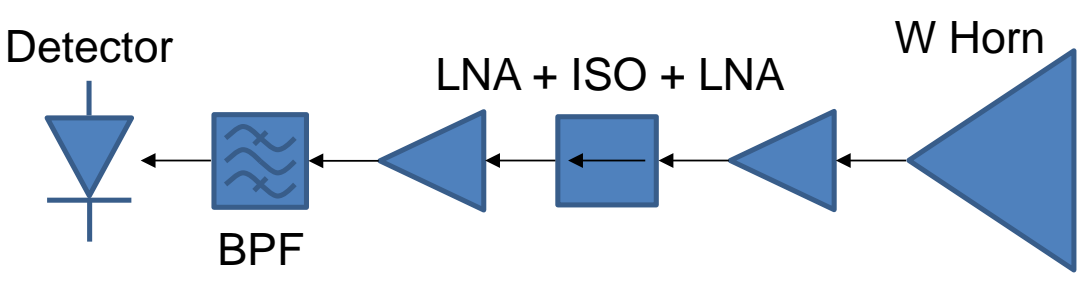

Hidden

L Shaped

Sample

Figure 5. Block diagram of the setup for passive measurements

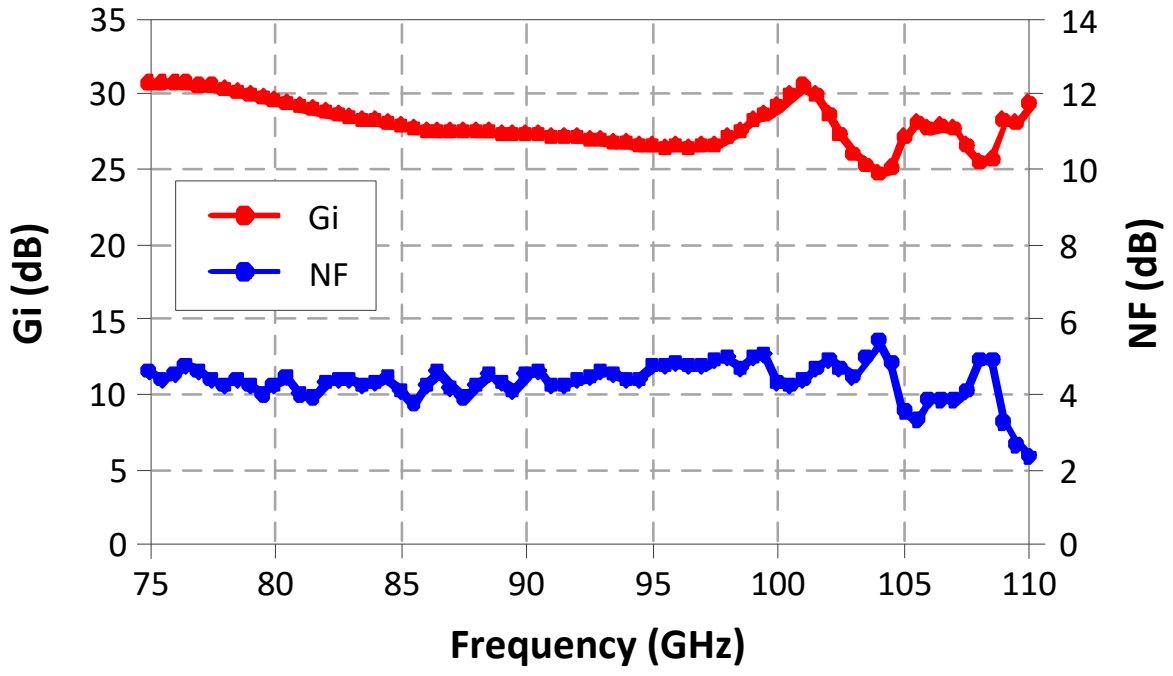

Figure 6. Measured noise figure \& insertion gain response of LNA5 in WR-10 case

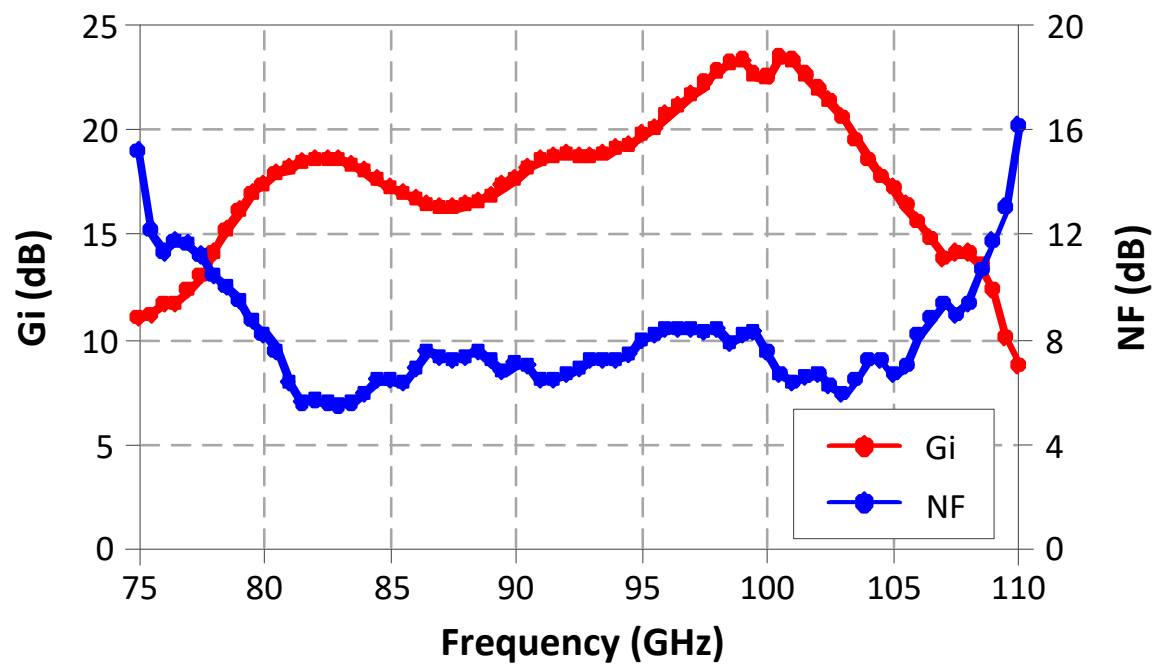

Figure 7. Measured noise figure \& insertion gain response of LNA4 in WR-10 case 


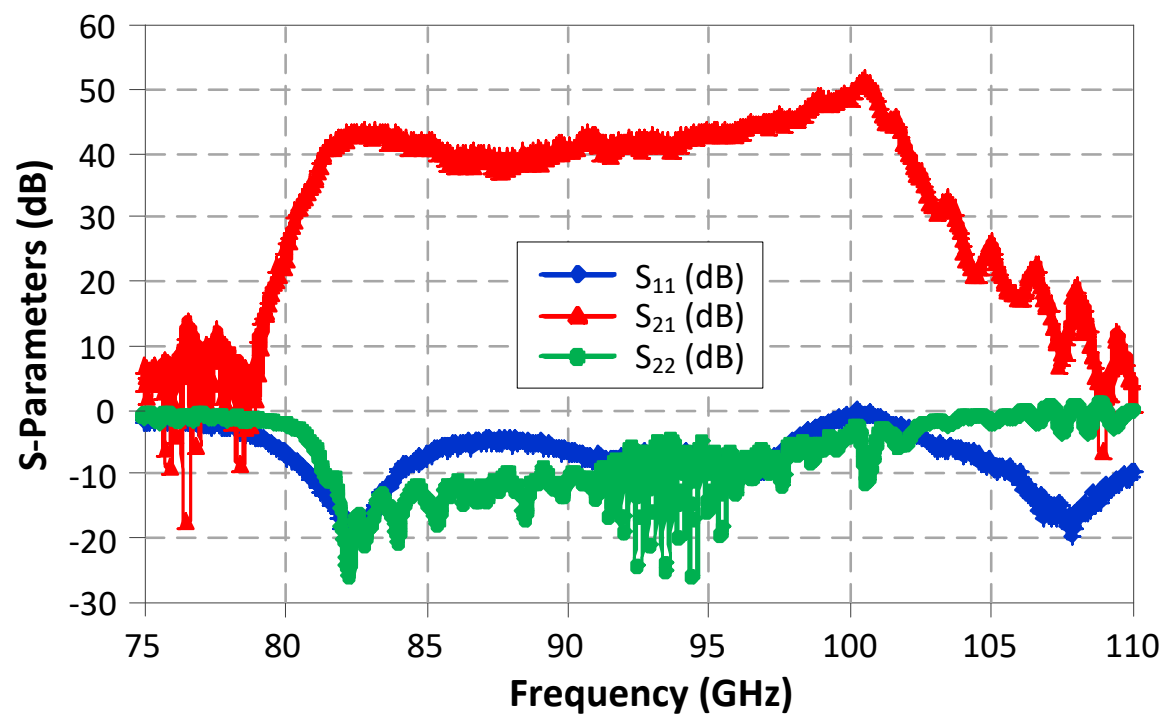

Figure 8. Measured Scattering response of the set: LNA4 + ISO + LNA5 + BPF

TABLE I. Effective noise bandwidth, room temperature input load and equivalent noise input power for the RF chain with and without band-pass filter, showing the effect of BPF, but also that the equivalent input noise level is in both cases higher than the input load level at room temperature

\begin{tabular}{cccc}
\hline RF Chain Elements & $\begin{array}{c}\mathrm{BW}_{\text {eff }} \\
(\mathrm{GHz})\end{array}$ & $\begin{array}{c}\mathrm{KToBW}_{\text {eff }} \\
(\mathrm{dBm})\end{array}$ & $\begin{array}{c}\mathrm{KTeBW}_{\text {eff }} \\
(\mathrm{dBm}) \\
(\text { estimated for NF=7dB) }\end{array}$ \\
\hline LNA4+ISO+LNA5 & 27.29 & -69.49 & -63.33 \\
LNA4+ISO+LNA5+BPF & 19.97 & -70.85 & -64.7 \\
\hline
\end{tabular}

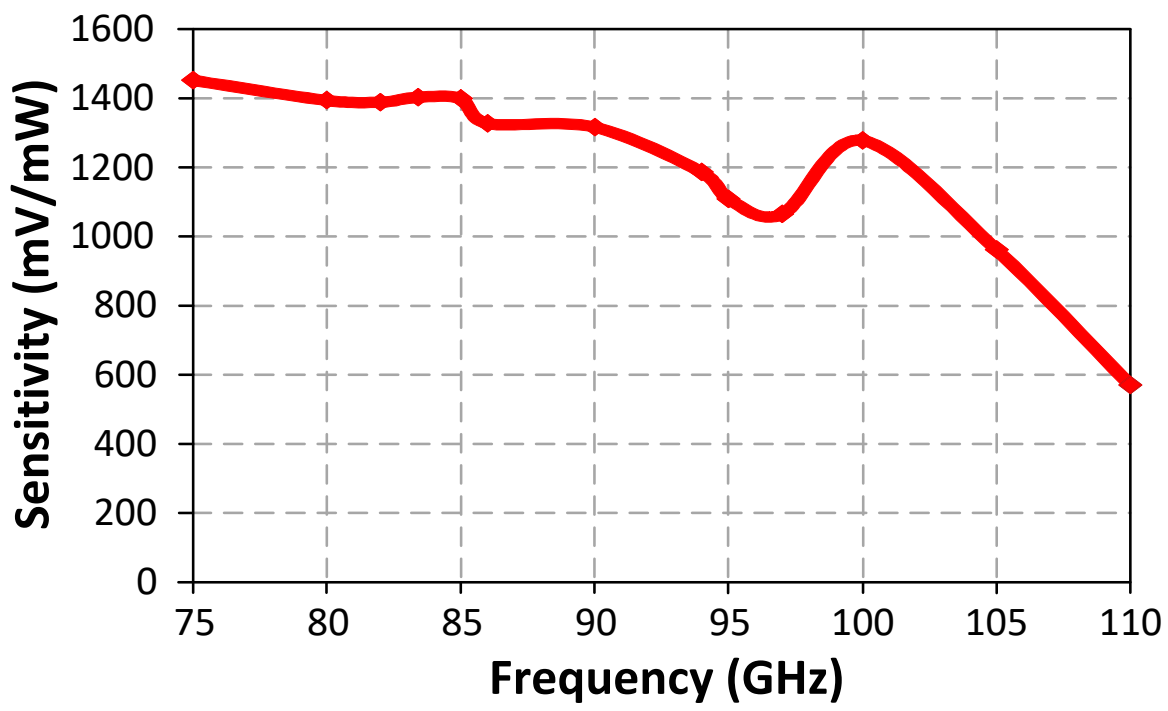

Figure 9. Measured detector response through the $\mathrm{W}$ band 


\section{B. Active Setups}

\section{B. 1 S $S_{11}$ mapping}

A basic active setup is configured with the mm-Wave head (manufacturer: OML) of a network analyzer. After one-port calibration, the horn antenna is connected to port 1 and a $S_{11}$ mapping can be obtained for the complete $\mathrm{W}$ band by sweeping in the whole range of the $\mathrm{x}-\mathrm{y}$ holder positions. This setup provides phase information. In Figure 10, a photo of the setup is shown, highlighting the $x-y$ sample holder with L shaped arm and micrometric screws.

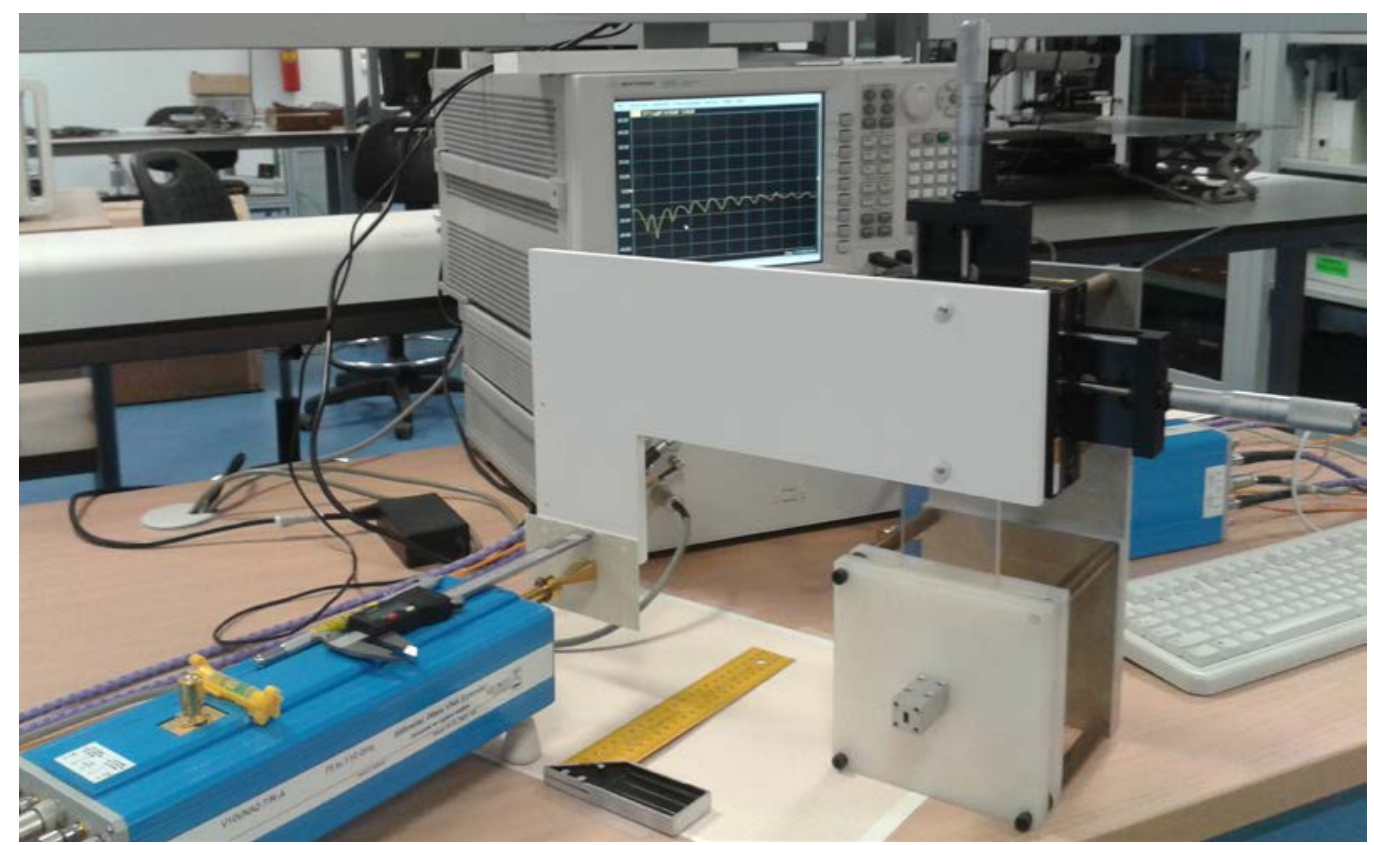

Figure 10. Photograph of the setup for $S_{11}$ mapping showing the $x-y$ sample holder

\section{B. 2 Detected voltage mapping with CW stimulus}

Another setup can be configured using the mm-Wave head as a W-band signal source operating in CW mode, connected to a circulator (model: ELVA1) and to the horn. The reflected signal passes back through the circulator (Figure 11 shows the circulator band-pass response between 90 and $100 \mathrm{GHz}$ ) and is detected in a W-band detector providing a dc value for each position, obtaining a detected voltage mapping for each CW frequency. Compared with the previous one, this setup does not provide phase information and restricts the band to the circulator response. In Figure 12 and Figure 13 the setup block diagram and a photograph are shown.

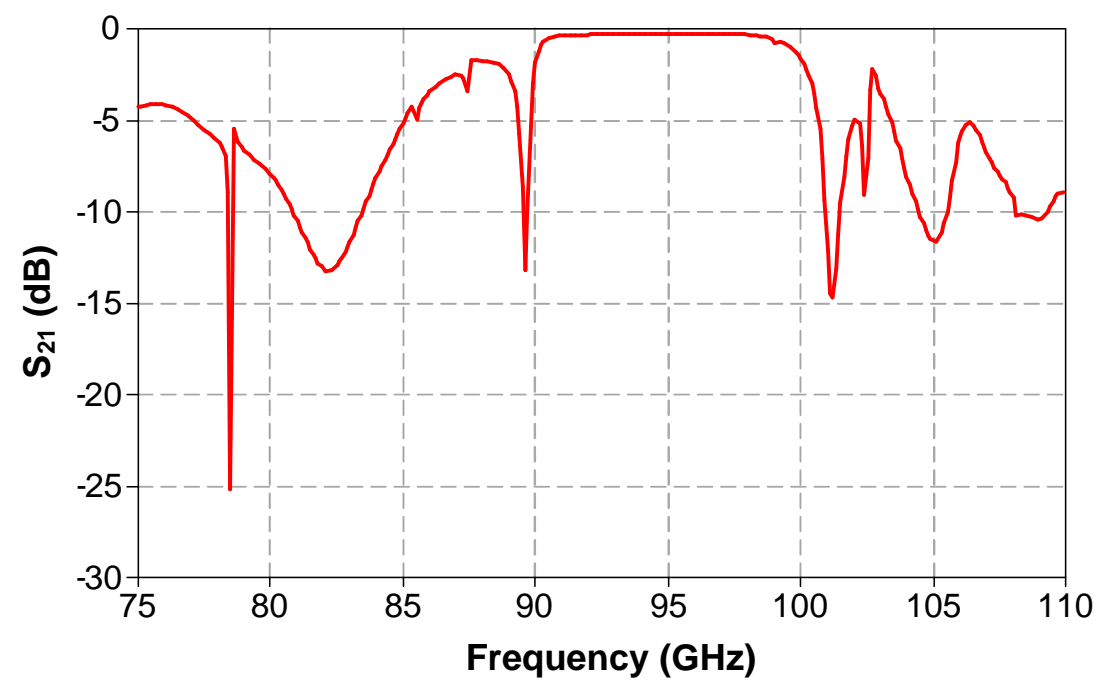

Figure 11. Circulator frequency response, limiting the operation band for $\mathrm{CW}$ active imaging 


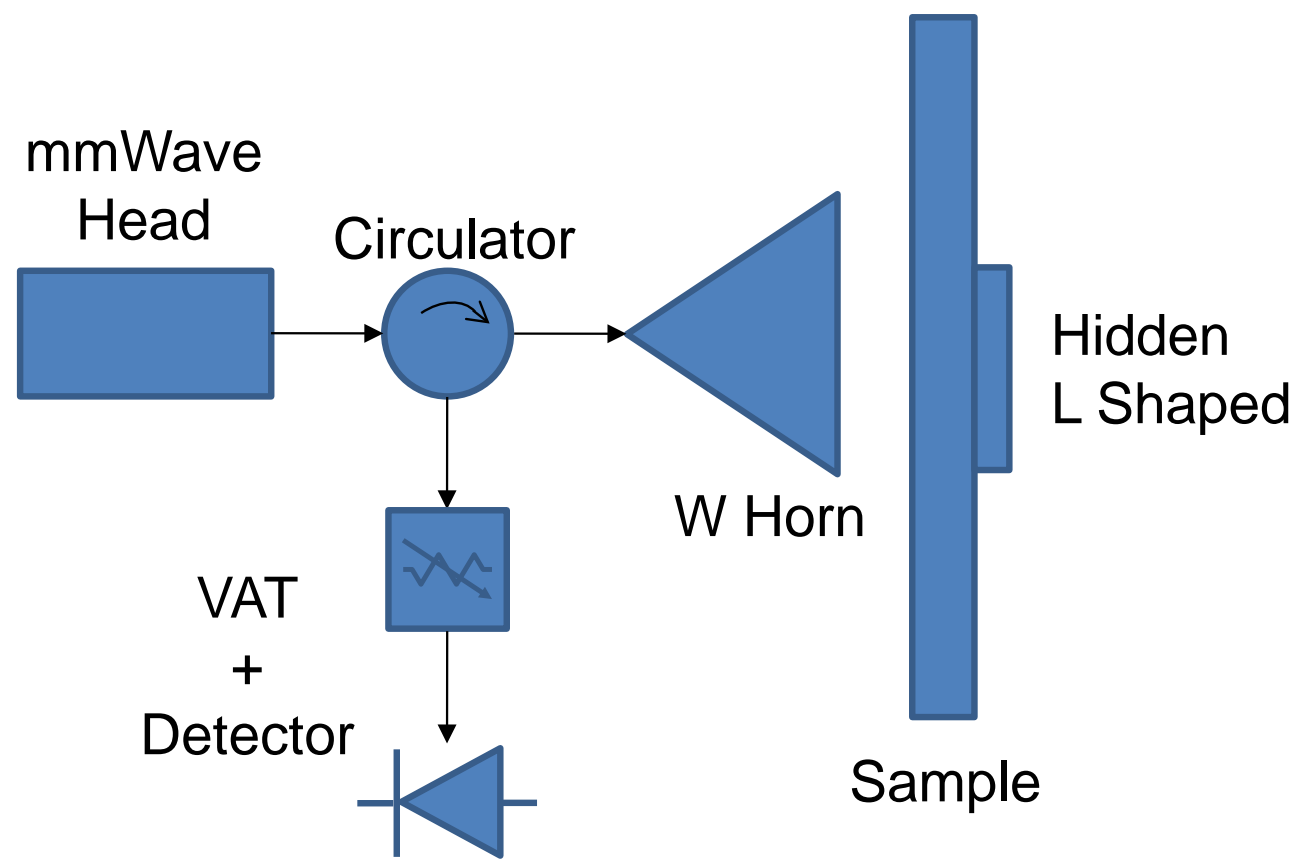

Figure 12. Block diagram of the setup for voltage mapping with CW stimulus and receiving the signal through a variable attenuator and a detector

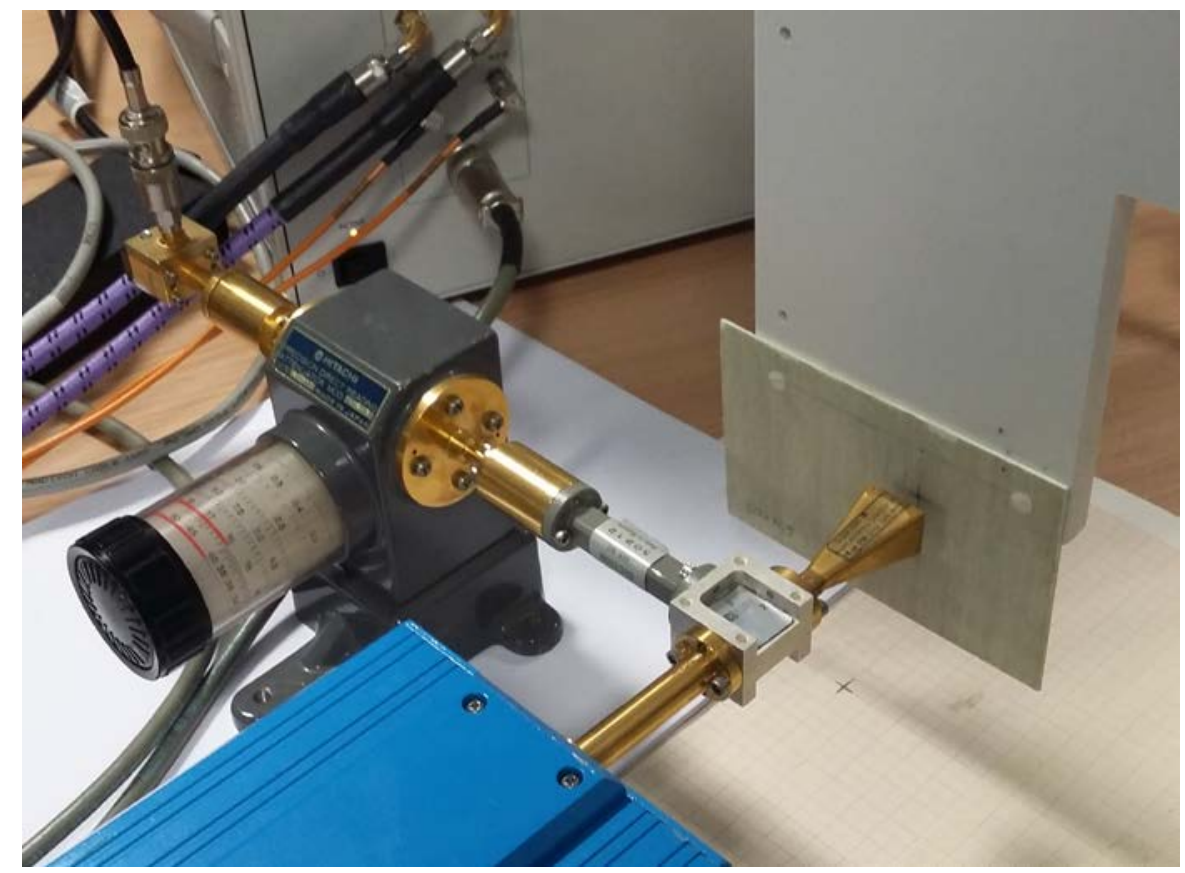

Figure 13. Photograph of the setup for voltage mapping showing the mmW head and the set of W-band components: circulator, horn in front of a sample, variable attenuator and detector

\section{B. 3 Detected voltage mapping with broadband noisy stimulus (illuminated passive system)}

Another setup can be configured using a W-band noise source (Quinstar) as W-band signal source operating in broadband mode, connected to a circulator and to the horn. The reflected signal passes back through the circulator and is amplified (two LNAs: LNA4 and LNA5 from HRL, with an isolator in between to reduce ripple), filtered (BPF) [18][19], if necessary, and finally detected by a W-band detector, providing a dc value for each position, obtaining a detected voltage mapping for the whole $\mathrm{W}$ frequency band. A variable attenuator (VAT) is included for safety, but finally set to $0 \mathrm{~dB}$. This setup does not provide phase information. It can be considered quite similar to the passive setup and suitable to 
emulate it. In fact, it could be defined as an "illuminated passive setup" rather than an active setup. The procedure flow diagram in this case would be quite similar to Figure 3, but using reflectivity from the EM simulations instead of emissivity. It should be noticed than a leakage contribution due to limited isolation of the circulator will be added to the incoming reflected signal causing an increase in the noise floor which could mean a loss of contrast. In Figure 14 and Figure 15 the setup block diagram and a photograph are shown, as well as the RF to dc response of the set for a sweeping tone, with or without BPF (Figure 16). In TABLE II, an estimation of the effective noise bandwidth for the RF chain with and without band-pass filter and with and without detector at the end is given. The corresponding load input power at room temperature, equivalent system noise power and noise power from noise source are also included, showing that in the active system, input power from noise source is greater than the system noise. The RF to dc system response when no filter is present shows a remarkable peak which should be taken into account further to evaluate the performance. Interaction between the RF chain response and detector response produces a reduction in effective bandwidth, but the dc response goes beyond the band defined by the filter and the circulator.

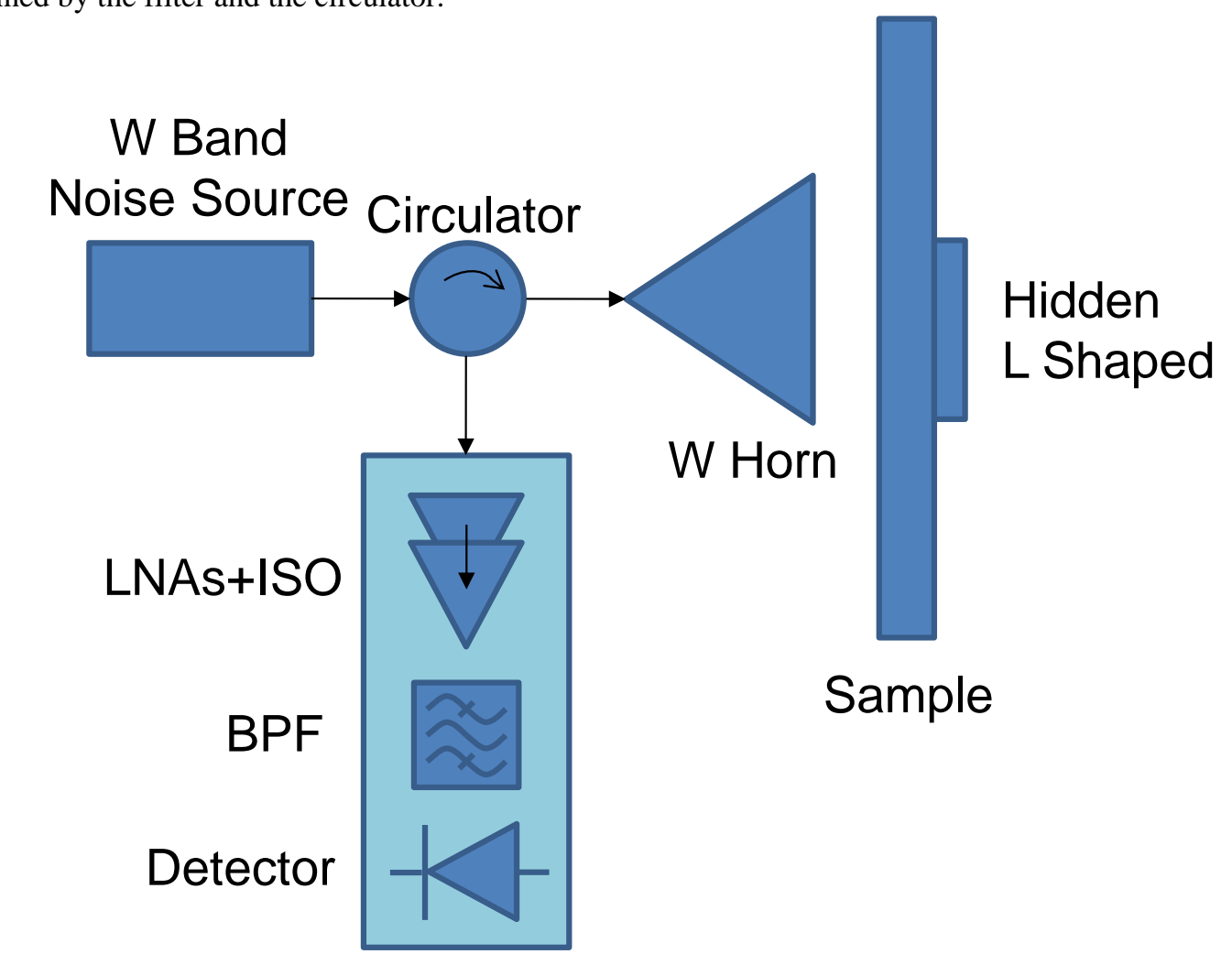

Figure 14. Block diagram of the setup for voltage mapping with broadband noise source stimulus. Images were obtained with and without band-pass filter (BPF) 


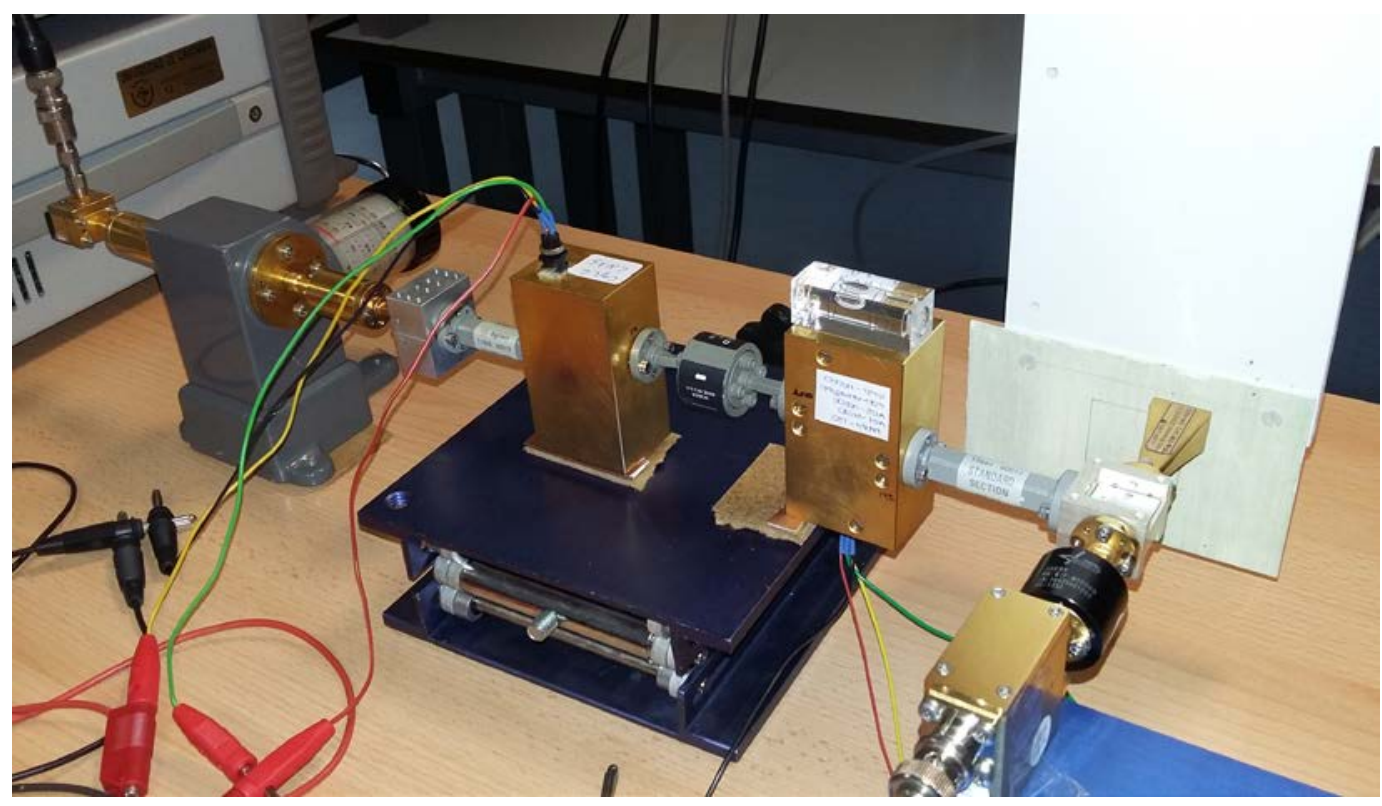

Figure 15. Photograph of the setup for voltage mapping showing W-band noise source and the set of W-band components: circulator, horn in front of sample, LNA4, isolator, LNA5, BPF, variable attenuator and detector

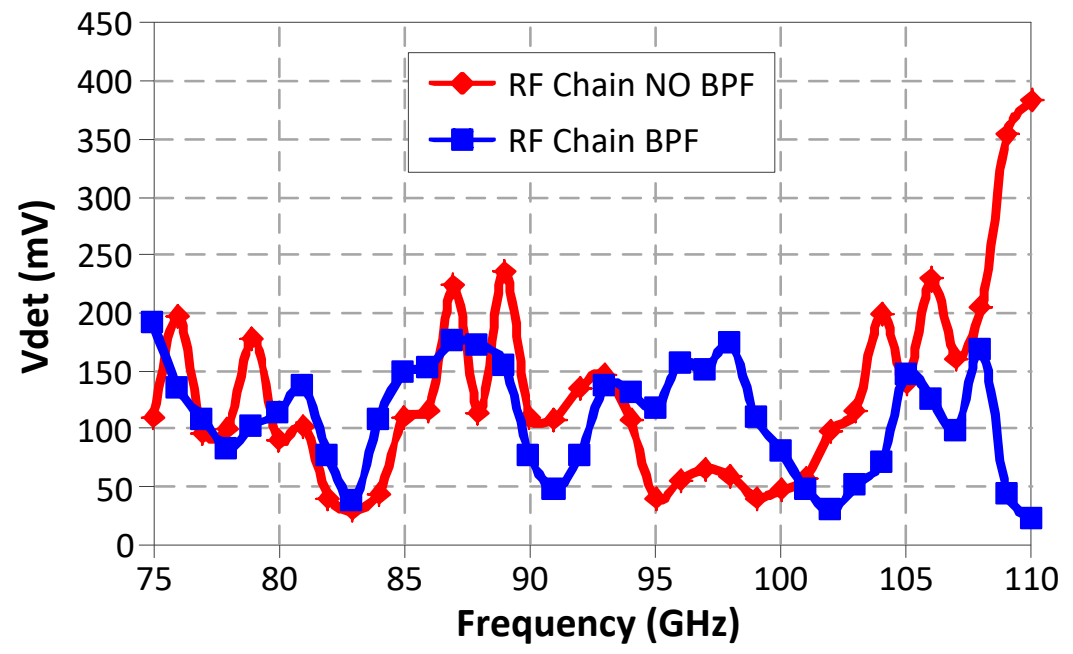

Figure 16. Characterization of the amplifying chain (with/without band-pass filter) in RF to DC for $-70 \mathrm{dBm}$ input power

TABLE II. Estimation of effective noise bandwidth for RF chain with and without band-pass filter and with and without detector at the end. Corresponding load input power at room temperature, equivalent system noise power and noise power from noise source are included

\begin{tabular}{ccccc}
\hline Chain Elements & $\begin{array}{c}\mathrm{BW}_{\text {eff }} \\
(\mathrm{GHz})\end{array}$ & $\begin{array}{c}\mathrm{KToBW}_{\text {eff }} \\
(\mathrm{dBm})\end{array}$ & $\begin{array}{c}\mathrm{KTeBW}_{\text {eff }} \\
(\mathrm{dBm}) \\
(\mathrm{NF} 7 \mathrm{~dB})\end{array}$ & $\begin{array}{c}\mathrm{KThBW}_{\text {eff }} \\
(\mathrm{dBm}) \\
(\mathrm{ENR} \mathrm{15} \mathrm{dB)})\end{array}$ \\
\hline ANT+CIRC+LNA4+ISO+LNA5 & 23.19 & -70.20 & -64.17 & -55.2 \\
ANT+CIRC+LNA4+ISO+LNA5+FPB & 17.23 & -71.49 & -65.46 & -56.49 \\
ANT+CIRC+LNA4+ISO+LNA5+DET & 14 & -72.39 & -66.36 & -57.39 \\
ANT+CIRC+LNA4+ISO+LNA5+FPB+DET & 10.19 & -73.54 & -67.74 & -58.77 \\
\hline
\end{tabular}




\section{RESULTS AND COMPARISONS}

Simulations were carried out of the mentioned sample (with a hidden L-shape behind a dielectric layer) scanned in both types of setups, passive and active (Scattering parameters, CW and noisy broadband). Measurements were completed for all the scenarios. In the case of a pure passive scenario, it was very difficult to achieve enough sensitivity to obtain a distinguishable image in an indoor environment with the two LNAs as an amplifying block before detection, as the figures in TABLE I predicted. Nevertheless, the detected voltage mapping with broadband noisy stimulus could be considered to be equivalent to an illuminated passive imaging system, and more feasible than a pure passive one. It provides a reasonable emulation of the passive scenario mainly in two aspects: the broadband features and the use of the radiometer block.

Figure 17 shows the hidden shape as a reference for comparison with images obtained by simulations and from measurements.

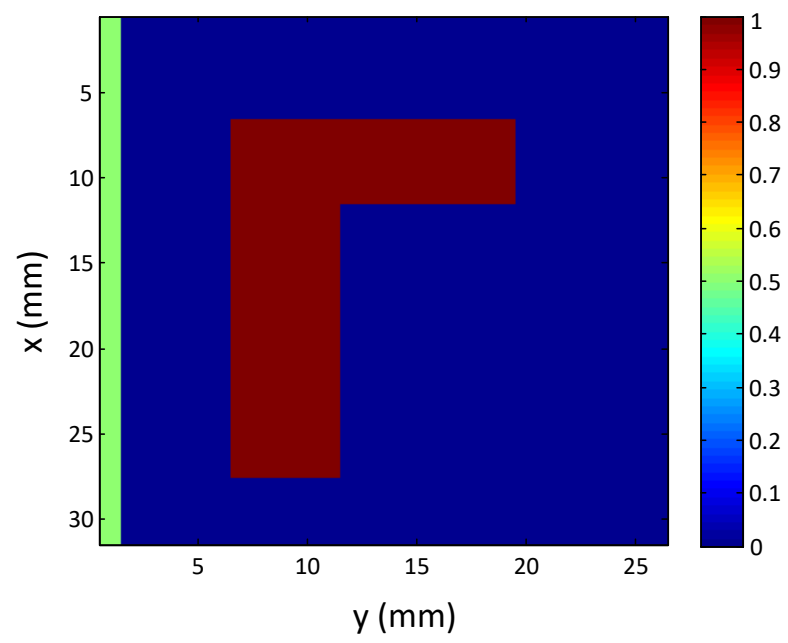

Figure 17. Hidden L-shape reference for comparison with images obtained by simulations and measurements (x and y coordinates in $\mathrm{mm}$ )

\section{A. Passive Setup}

Following the described procedure for 3D EM simulations and the post-processing of data using MatLab [20] and a circuit-system simulator [15] the following results were obtained by simulation for the case of a hidden L-shaped layer of a different dielectric. Values are averaged in the whole $\mathrm{W}$ band. System sensitivity becomes a critical factor in this pure passive setup. A $3 \mathrm{~dB}$ noise figure (288 K) was assumed for the simulations corresponding to these plots. Higher noise figures make the image more undistinguishable. It should be noticed that in this deterministic simulation the voltage range is very narrow, about $3 \mathrm{mV}$, which leads to great difficulties to obtain an image from a practical system. 


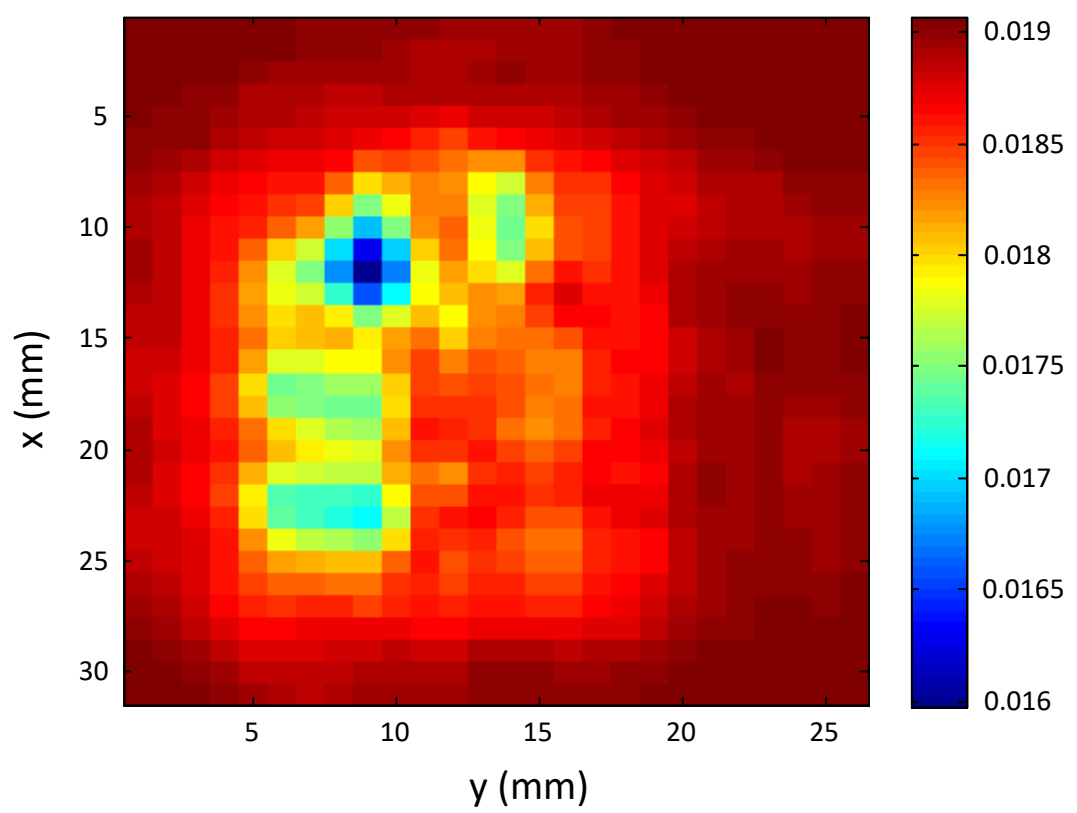

Figure 18. Image of the hidden L-shape with a different dielectric obtained by simulation averaging in the whole W band ( $x$ and $y$ coordinates in $\mathrm{mm}$ )

When the image of the hidden L-shape with a different dielectric is averaged in the 97-110 GHz band better contrast is obtained, compared to full $\mathrm{W}$ band as can be seen in the Figure 19 .

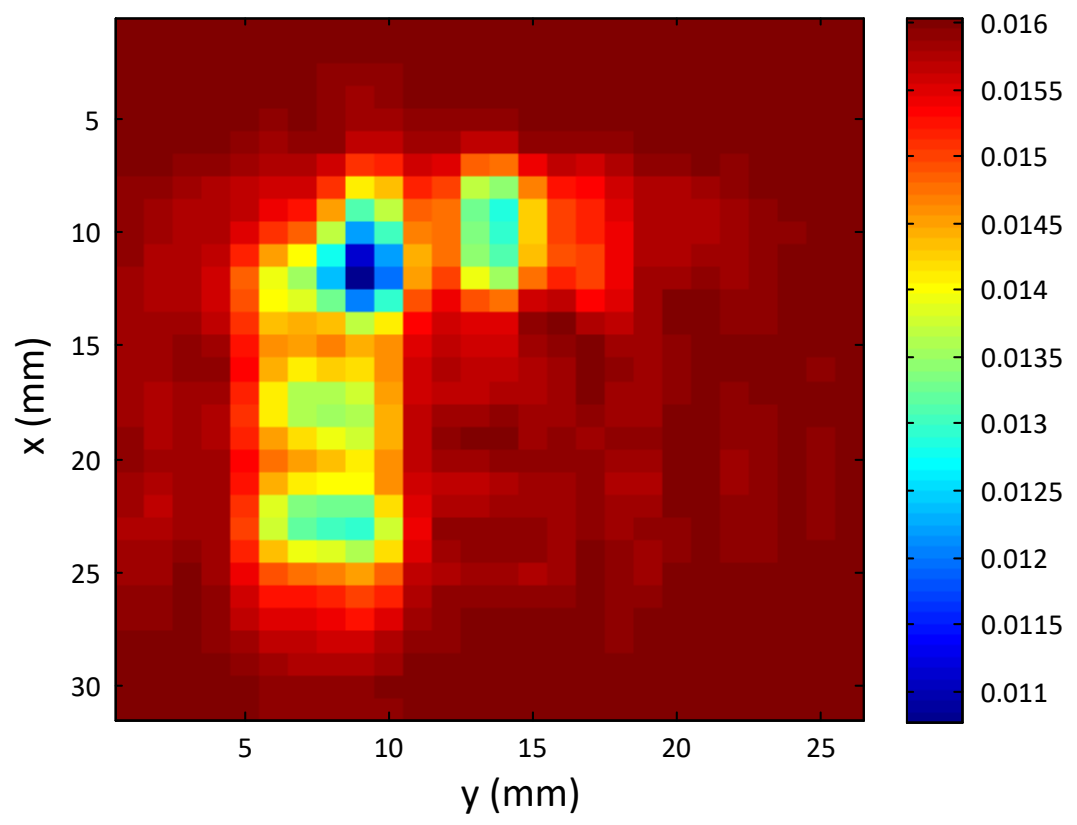

Figure 19. Image of the hidden L-shape with a different dielectric obtained by simulation averaging in the 97-110 GHz band ( $\mathrm{x}$ and y coordinates in $\mathrm{mm}$ )

According to simulations in the pure passive setup some band conformation could be useful to improve the image, in contrast to the concept of the wider the band, the better the image. Nevertheless the most worrying aspect is the narrow voltage range.

As was mentioned, in this case, with the initial setup, there are no useful measurements available. Practical tests showed the difficulty in achieving a sufficient voltage level difference corresponding to power level difference that is enough to excite the detector in an indoor environment. It should be considered that the two LNAs before detection provided a noise figure higher than expected (about 5/7 $\mathrm{dB}$ ). Another LNA manufactured by OMMIC with lower noise figure (about $3.5 \mathrm{~dB}$ ) was added in front of the chain to provide better sensitivity. Nevertheless, the following image was obtained: 


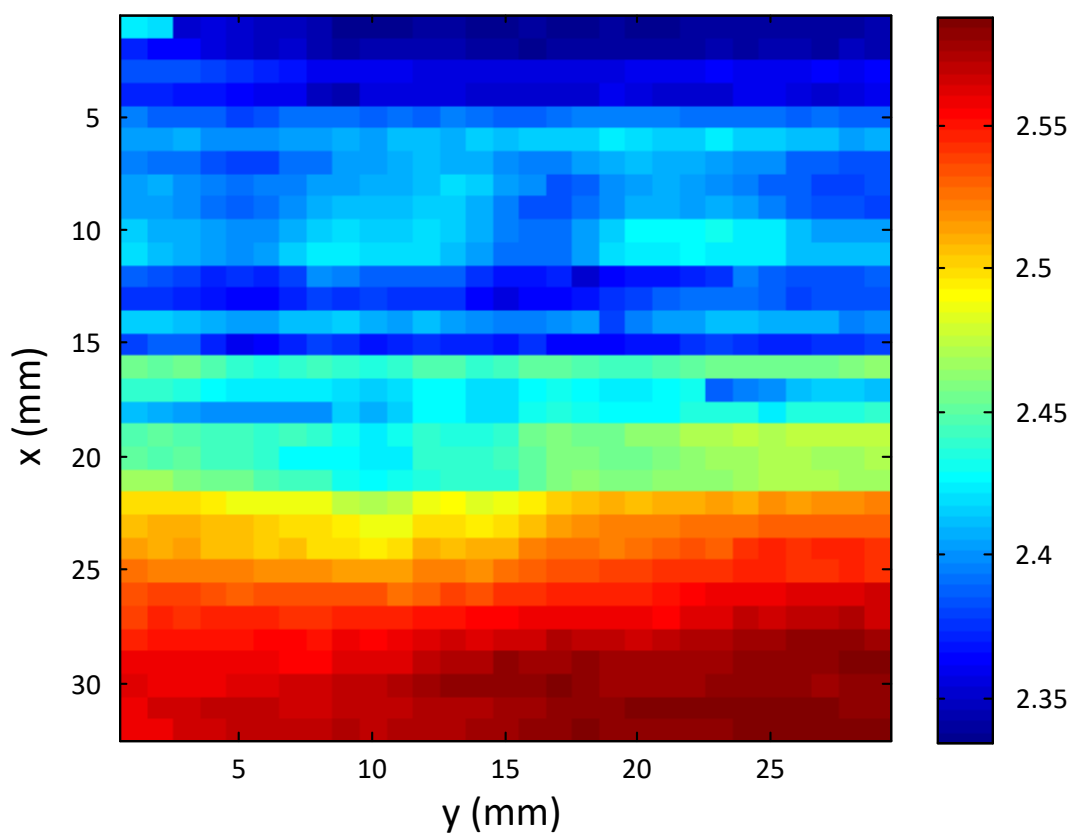

Figure 20. Measured image of the hidden L-shape obtained with a radiometer chain with an additional LNA to decrease the noise figure. As it can be seen, the noise figure decrement was not enough to get a useful image. Output voltage was DC amplified (x and y coordinates in $\mathrm{mm}$ )

The addition of another LNA to the chain was not successful, insufficiently decreasing the noise figure, and also narrowing the system's dynamic range, as LNAs may eventually compress. Therefore, due to the lack of lower sensitivity LNAs, illumination was required to reach sufficient power level differences to be detected. This finding is in agreement with [3] where passive sensing is applied with illumination or in an outdoor environment. An increase in the integration level may help to limit the losses in radiometers [21].

\section{B. Active Setups}

Three types of active setups were distinguished: based on $S_{11}$ mapping and based on detected voltage mapping with CW stimulus and with broadband noisy stimulus (illuminated passive system).

\section{B. $1 \quad S_{11}$ mapping}

Images were obtained measuring the $S_{11}$ parameter at a fixed frequency and also averaging over a frequency range. The sample with a hidden L-shaped metal layer and the sample with a hidden L-shaped layer with a different dielectric $\left(\varepsilon_{\mathrm{r}}=10\right.$ behind $\left.\varepsilon_{\mathrm{r}}=3.48\right)$ were used to compare the ability to provide images, not only of hidden metal objects, but also of different dielectric materials.

Measurements of $\mathrm{S}_{11}$ in magnitude at $90 \mathrm{GHz}$ of a hidden L-shaped metal layer are shown in Figure 21. 


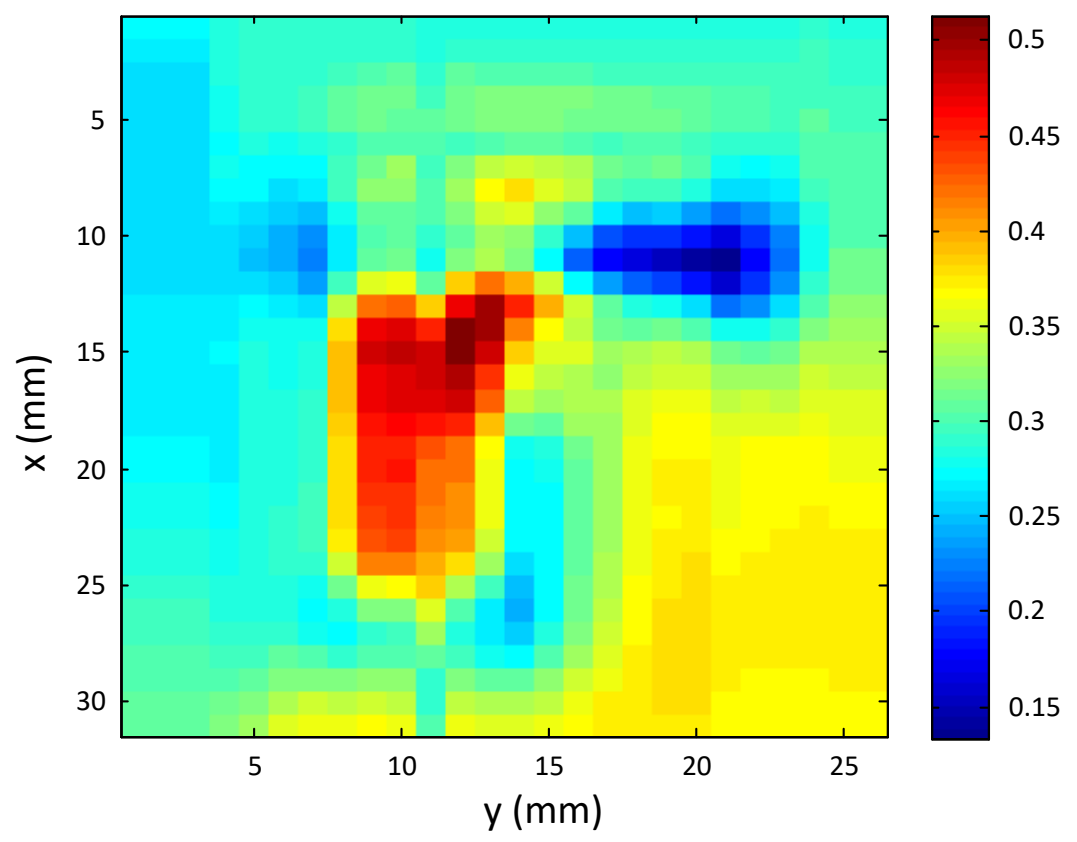

Figure 21. Measured image of $S_{11}$ in magnitude at $90 \mathrm{GHz}$ of hidden L-shaped metal layer (x and y coordinates in mm)

At a frequency of $94 \mathrm{GHz}$, the $\mathrm{S}_{11}$ magnitude image of the hidden L-shaped metal layer is obtained and shown in Figure 22.

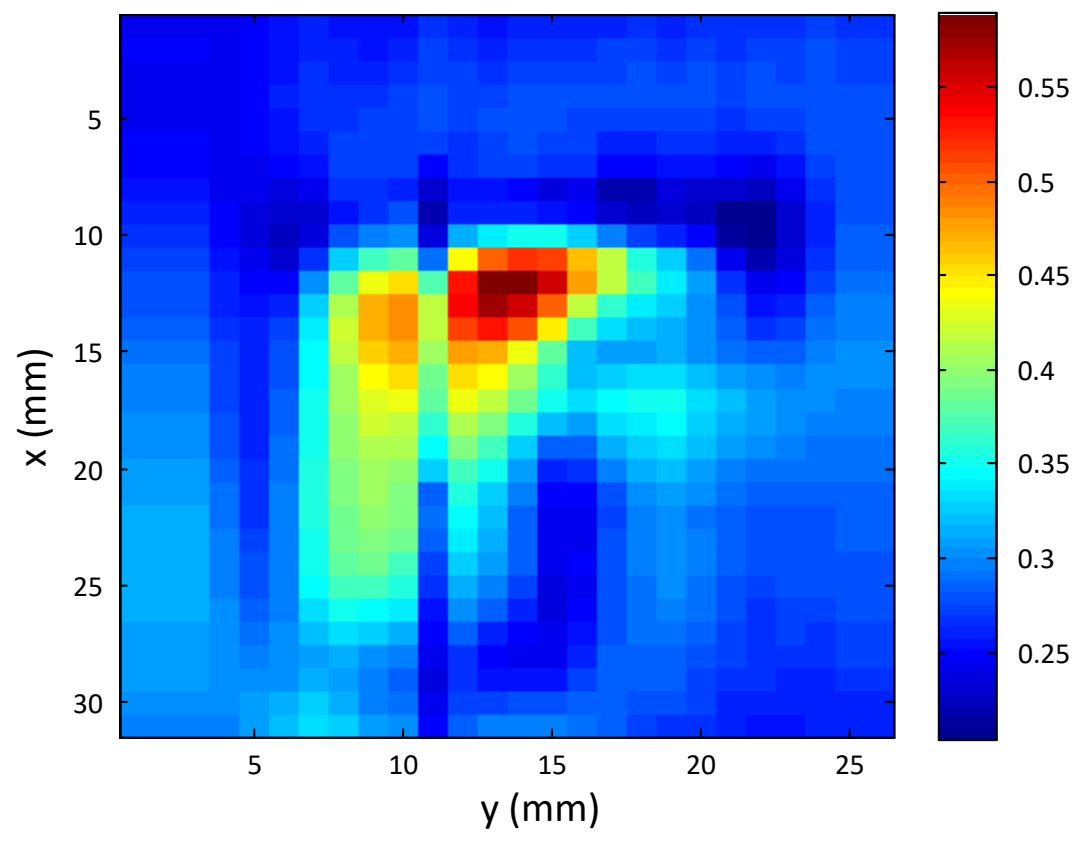

Figure 22. Measured image of $S_{11}$ in magnitude at $94 \mathrm{GHz}$ of hidden L-shaped metal layer ( $\mathrm{x}$ and y coordinates in $\mathrm{mm}$ )

Measurements of $S_{11}$ in magnitude at $90 \mathrm{GHz}$ of a hidden L-shaped layer with a different dielectric are depicted in Figure 23 showing a similar shape to the one obtained with hidden metal. 


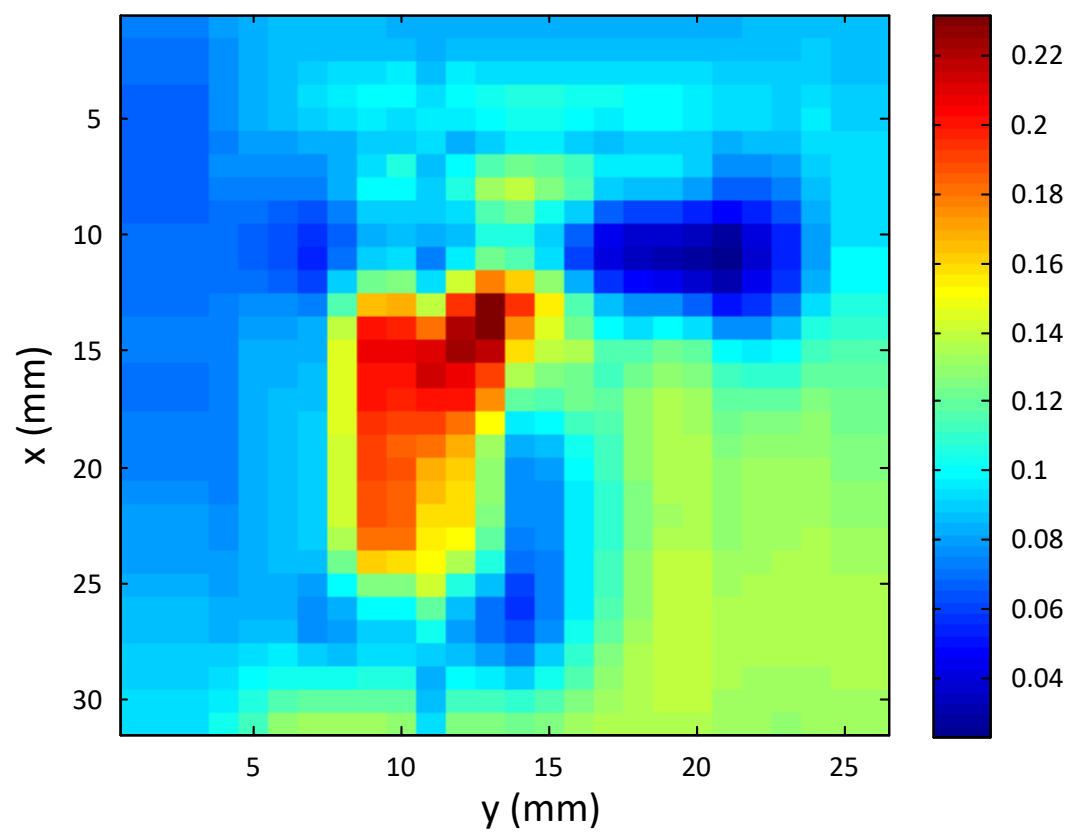

Figure 23. Measured image of $S_{11}$ in magnitude at $90 \mathrm{GHz}$ of hidden L-shaped layer with a different dielectric (x and y coordinates in $\mathrm{mm}$ )

At a frequency of $94 \mathrm{GHz}$, the $\mathrm{S}_{11}$ magnitude image of the hidden L-shaped layer with a different dielectric is obtained and shown in Figure 24. In this case, there is a big difference compared to the shape obtained with hidden metal.

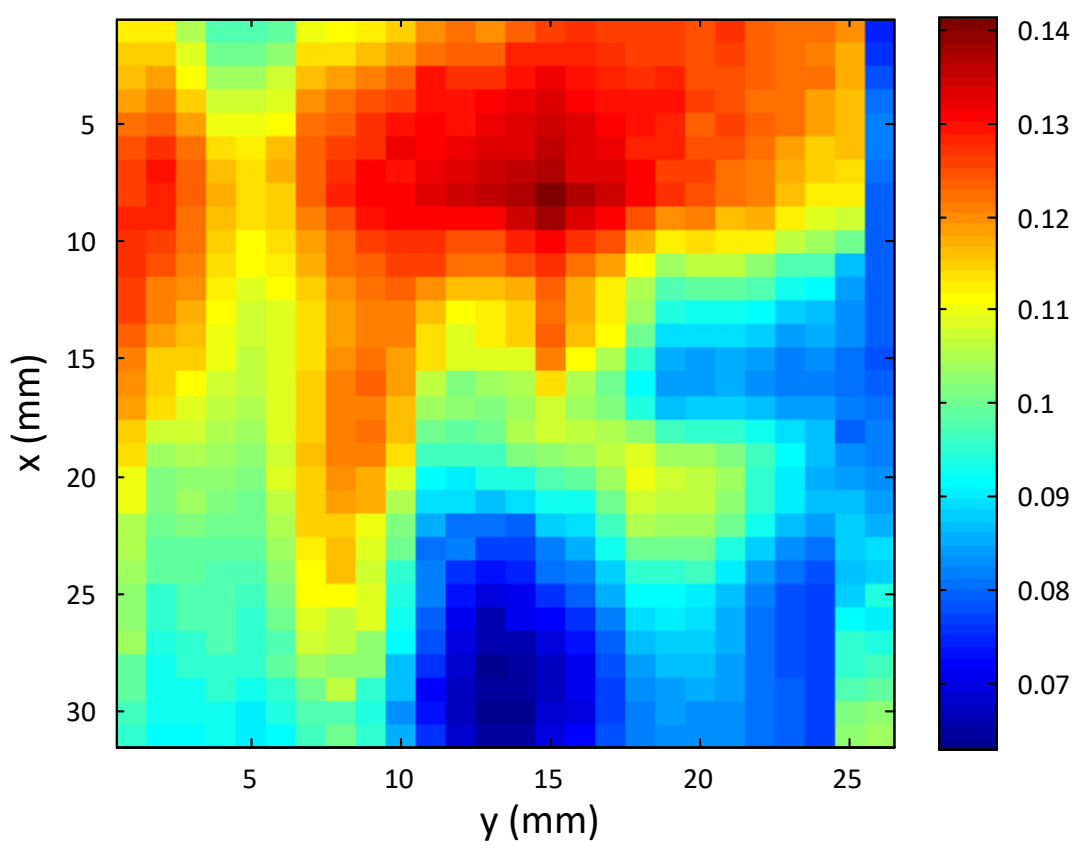

Figure 24. Measured image of $\mathrm{S}_{11}$ in magnitude at $94 \mathrm{GHz}$ of hidden L-shaped layer with a different dielectric (x and y coordinates in $\mathrm{mm}$ )

In general, there are available reconstruction algorithms like [12] and deblurring algorithms [20] to reconstruct and improve image quality, but our setting is not well suited to apply them due to the actual too short ranges. Considering the squared magnitude, $\left[S_{11}\right]^{2}$ averaged over the $90-100 \mathrm{GHz}$ range of the hidden L-shaped layer with a different dielectric, the image obtained is shown in Figure 25. 


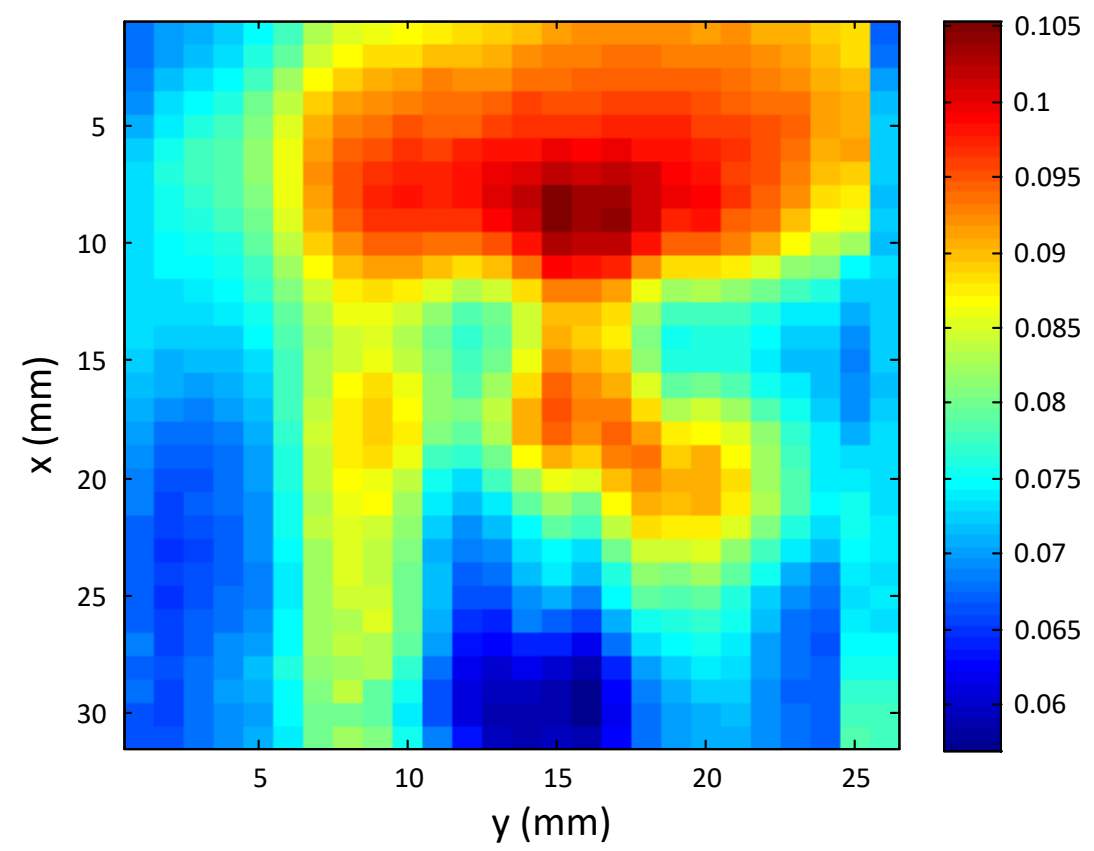

Figure 25. Measured image obtained from squared magnitude $\left[\mathrm{S}_{11}\right]^{2}$ averaged over the $90-100 \mathrm{GHz}$ range of a hidden L-shaped layer with a different dielectric ( $\mathrm{x}$ and y coordinates in $\mathrm{mm}$ )

If the image of the hidden L-shaped metal layer is obtained by averaging squared magnitude $\left[\mathrm{S}_{11}\right]^{2}$ over the 75-100 GHz range, the result is shown in Figure 26.

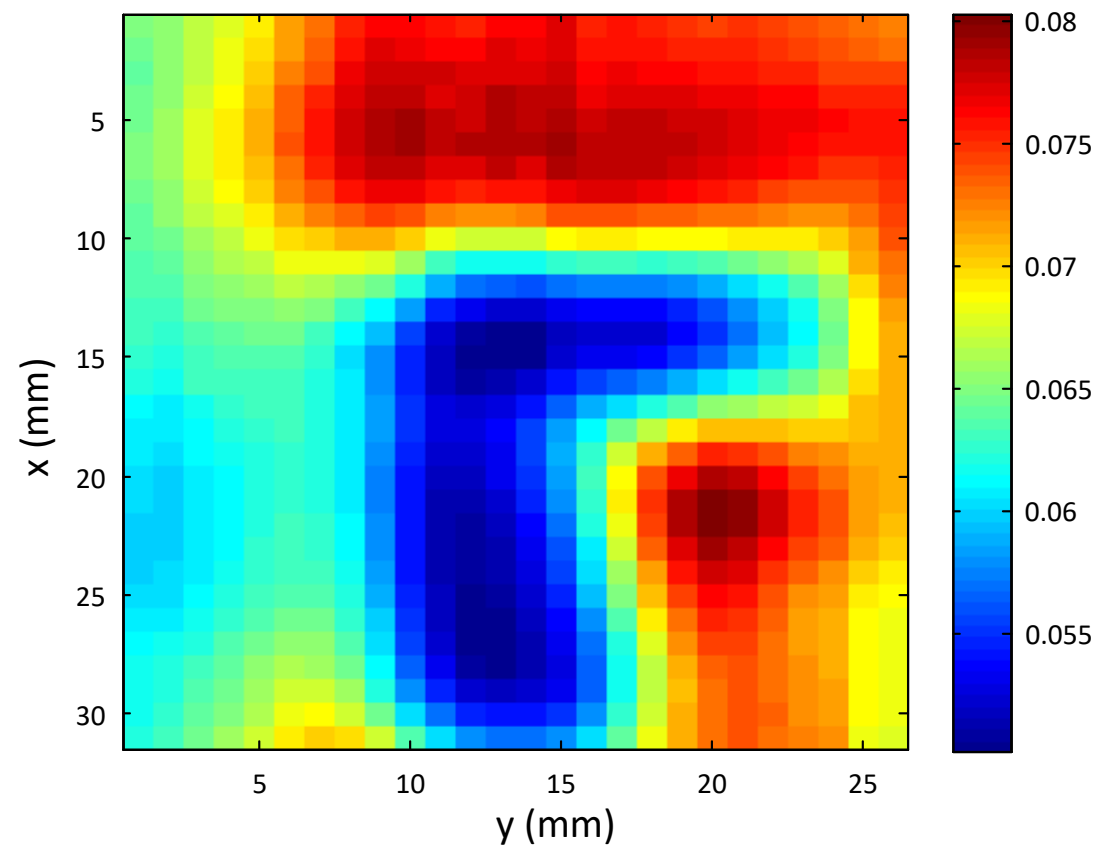

Figure 26. Measured image obtained from squared magnitude $\left[\mathrm{S}_{11}\right]^{2}$ averaged over the $75-100 \mathrm{GHz}$ range of hidden L-shaped layer with a different dielectric ( $\mathrm{x}$ and $\mathrm{y}$ coordinates in $\mathrm{mm}$ )

This experiment was useful to study the resolution of the setup, by sweeping the "y" dimension (dotted line in Figure 27) at a fixed $x$ and observing the variation in magnitude of $S_{11}$. 


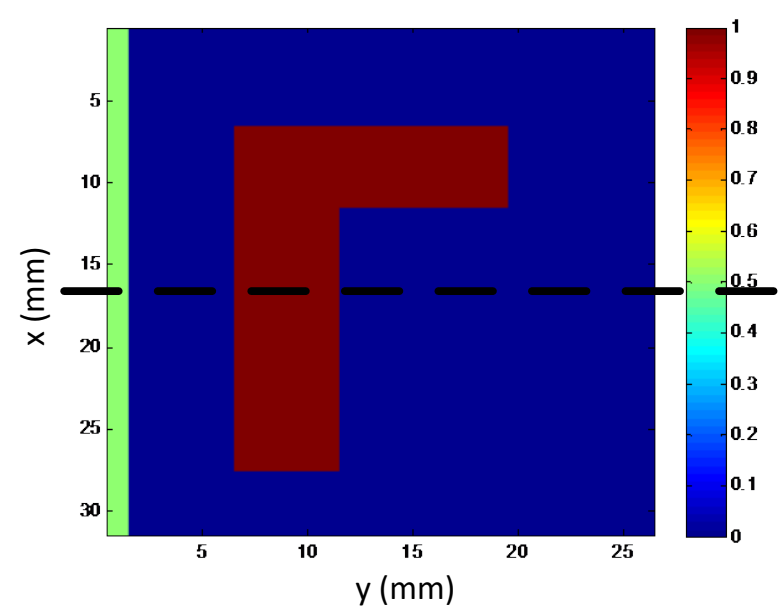

Figure 27. Representation of coordinates for variation of $S_{11}$ versus y position for a fixed x position. Hidden $\mathrm{L}$ shape is 5 mm wide ( $\mathrm{x}$ and $\mathrm{y}$ coordinates in $\mathrm{mm}$ )

Measurements of $\mathrm{S}_{11}$ sweeping "y" position for a fixed "x" in the W band were made showing the profile in Figure 28.

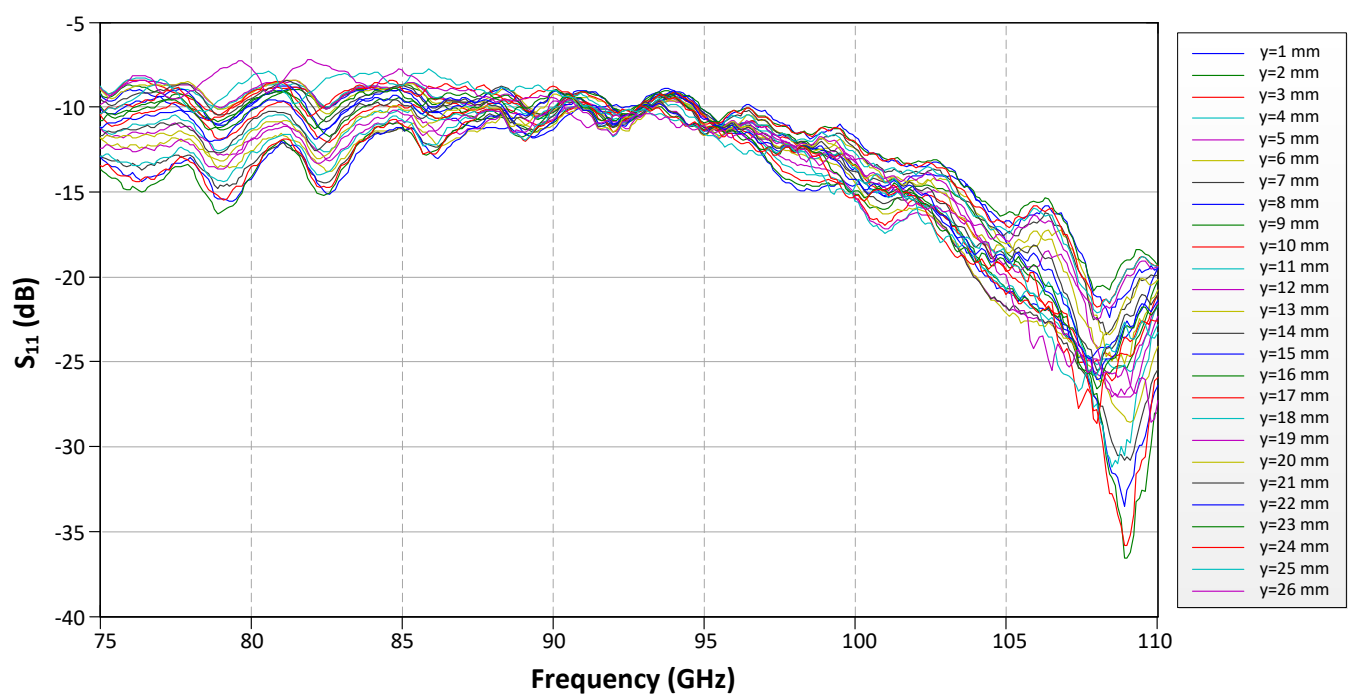

Figure 28. Measured $S_{11} d B$ versus frequency for several y positions at a fixed $x$

The same scene has been simulated with 3D EM simulations. First of all, the scene without sample target (basically horn input matching) is simulated and compared with measurements to validate coherence of the procedure, showing a good agreement (see Figure 29). Then, with the sample placed in its position, $\mathrm{S}_{11}$ was simulated sweeping y position for a fixed $\mathrm{x}$ in the whole $\mathrm{W}$ band (see Figure 30 ). 


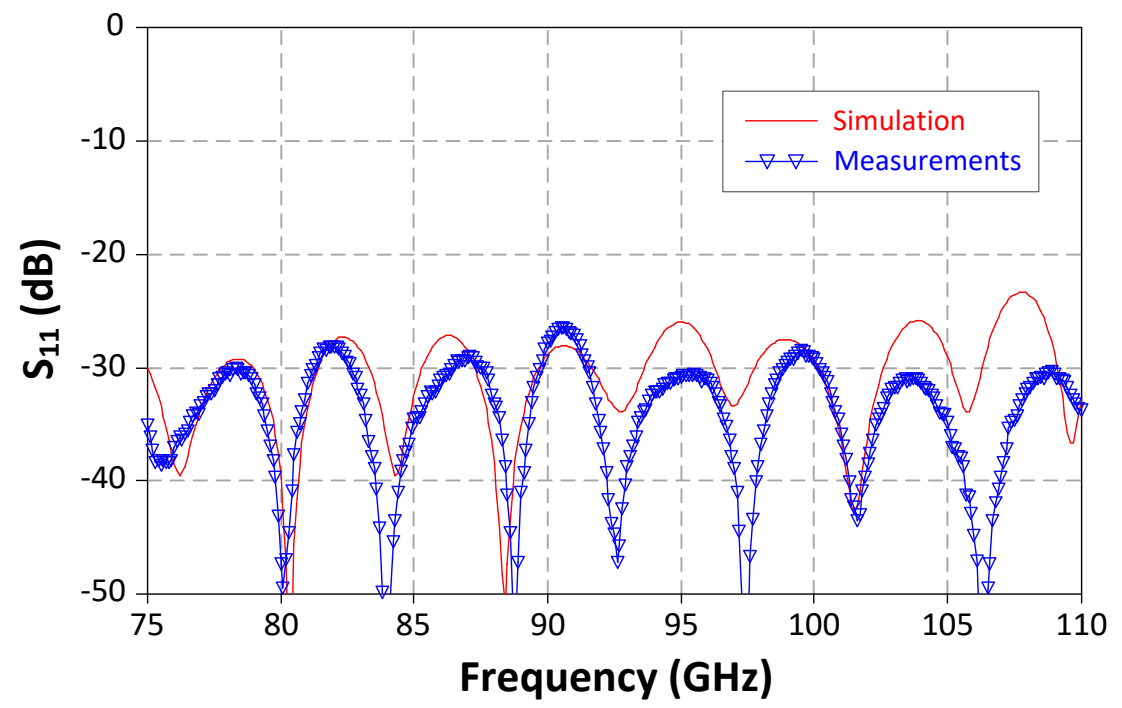

Figure 29. $\mathrm{S}_{11}$ in $\mathrm{dB}$ measured (blue triangles) and simulated (red line) versus frequency without sample target (horn input matching)

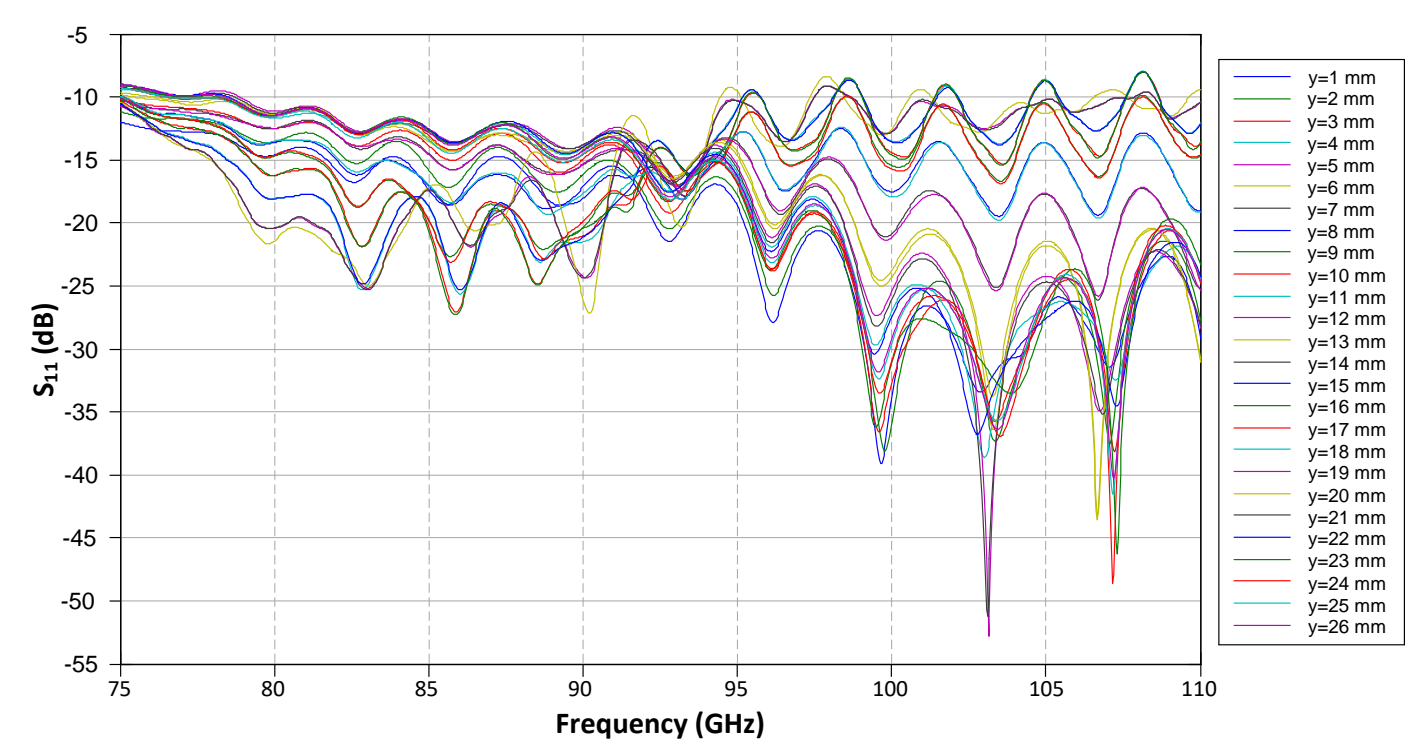

Figure 30. Simulated $S_{11} d B$ versus frequency for several y positions at a fixed $x$

Figure 28 and Figure 30 can be compared as measurements and simulations of the same scene for various y positions across the $\mathrm{W}$ band range. The measured variation shows less sensitivity in the range around $92.5 \mathrm{GHz}$ with the actual setup, matching with simulated results. In other frequency ranges (around $80-85 \mathrm{GHz}$ and around $105-107 \mathrm{GHz}$ ) simulations predict higher variation of $\mathrm{S}_{11}$ with "y" position than measured. Among other reasons, dielectric losses model and edge effects in a non-anechoic measurement environment may contribute to the differences between measurements and simulations.

Variation of $\mathrm{S}_{11}$ versus "y" position (for fixed "x") provides spatial resolution data at different frequencies (data were collected with $\Delta \mathrm{y}=1 \mathrm{~mm}$ ). In figures $31, \mathrm{~S}_{11} \mathrm{~dB}$ versus "y" position mm measured (a) and simulated (b) at $80 \mathrm{GHz}$ and their derivatives measured (c) and simulated (d) are plotted. Results show the setup limitation in resolving a hidden L-shape of $5 \mathrm{~mm}$ width. 


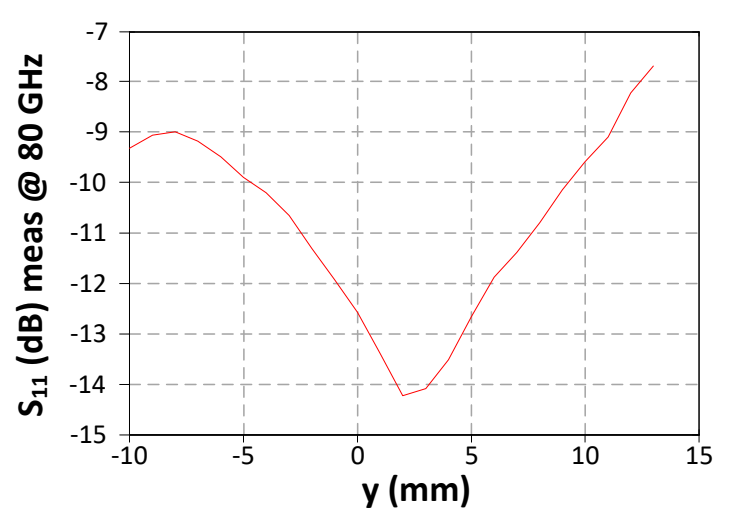

(a)

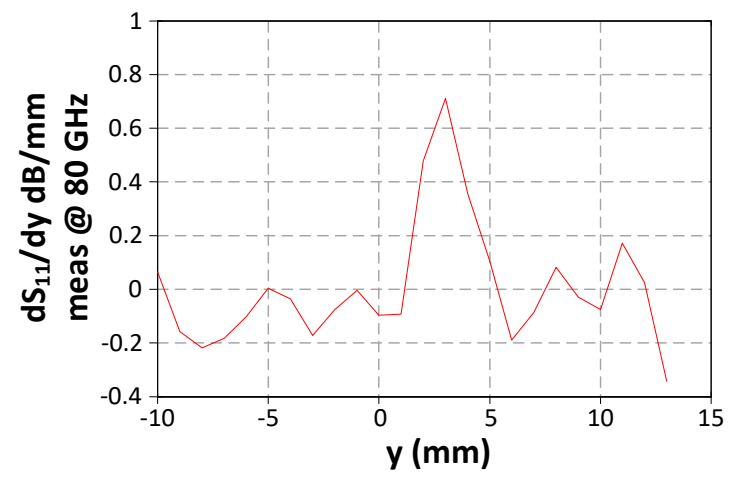

(c)

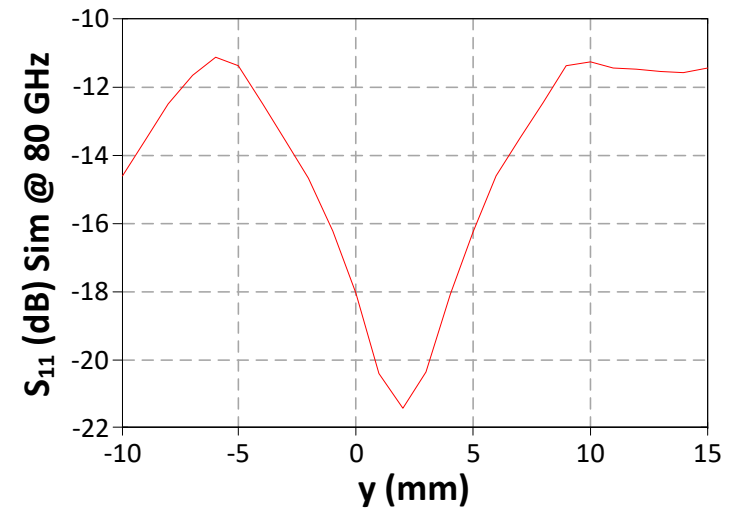

(b)

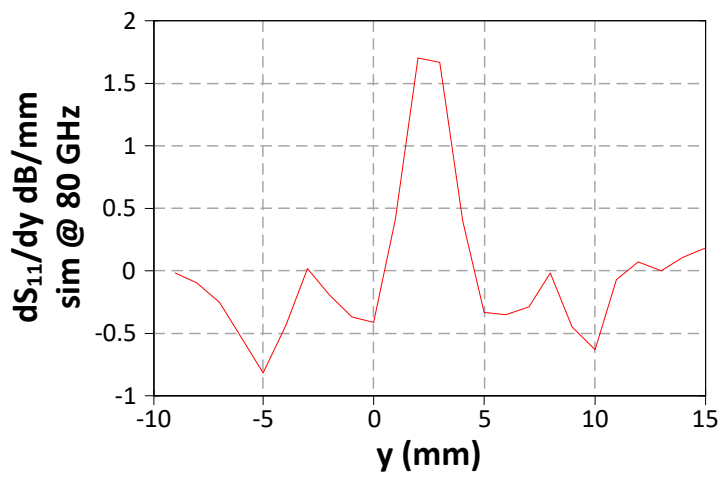

(d)

Figure 31. $\mathrm{S}_{11} \mathrm{~dB}$ versus “y” position mm measured (a) and simulated (b) at $80 \mathrm{GHz}$ and their derivatives measured (c) and simulated (d)

\section{B. 2 Detected voltage mapping with CW stimulus}

Images were obtained measuring detected voltage with a fixed frequency stimulus. Frequencies of 90 $\mathrm{GHz}, 94 \mathrm{GHz}$ and $97 \mathrm{GHz}$ were used for comparison. In Figure 32, measured voltages with CW stimulus at $90 \mathrm{GHz}$ (a), $94 \mathrm{GHz}$ (b) and at $97 \mathrm{GHz}$ (c) of the hidden L-shaped metal layer are shown. 94-GHz and 97-GHz images resemble better the reference shape. Particularly, the 94-GHz image is quite similar to the one obtained with $\mathrm{S}_{11}$ mapping (Figure 22).

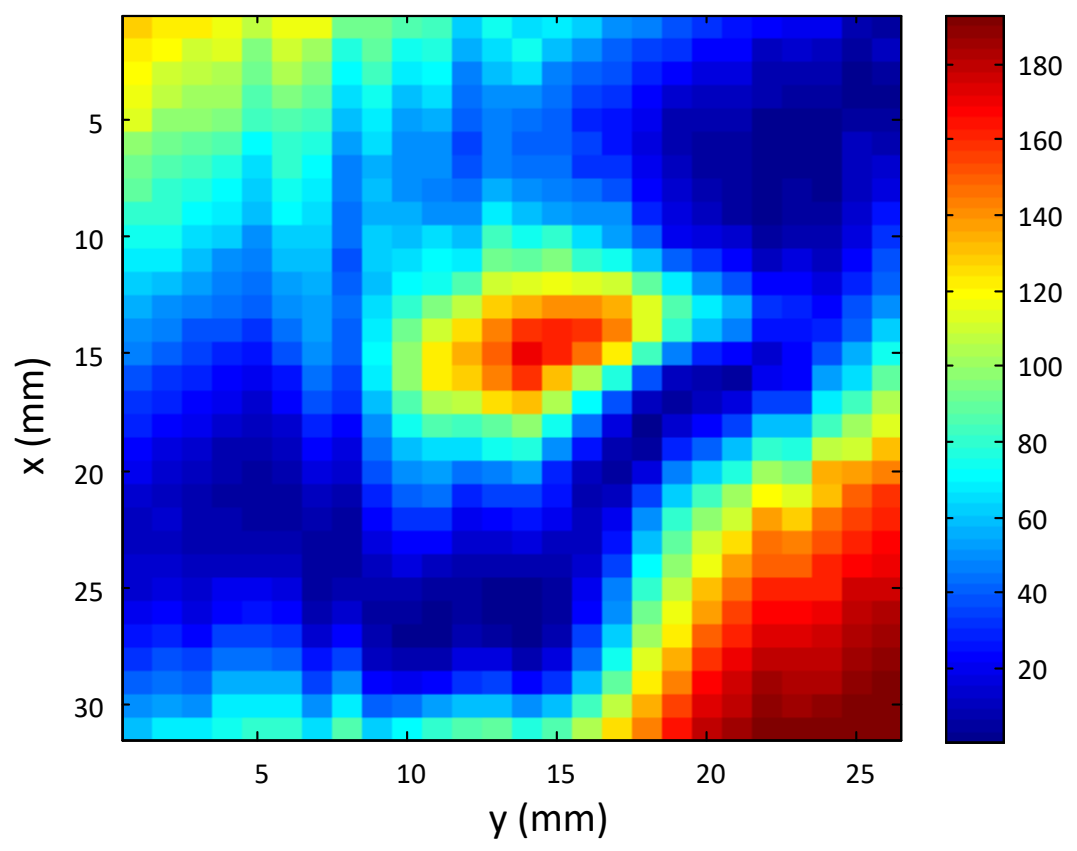


(a)

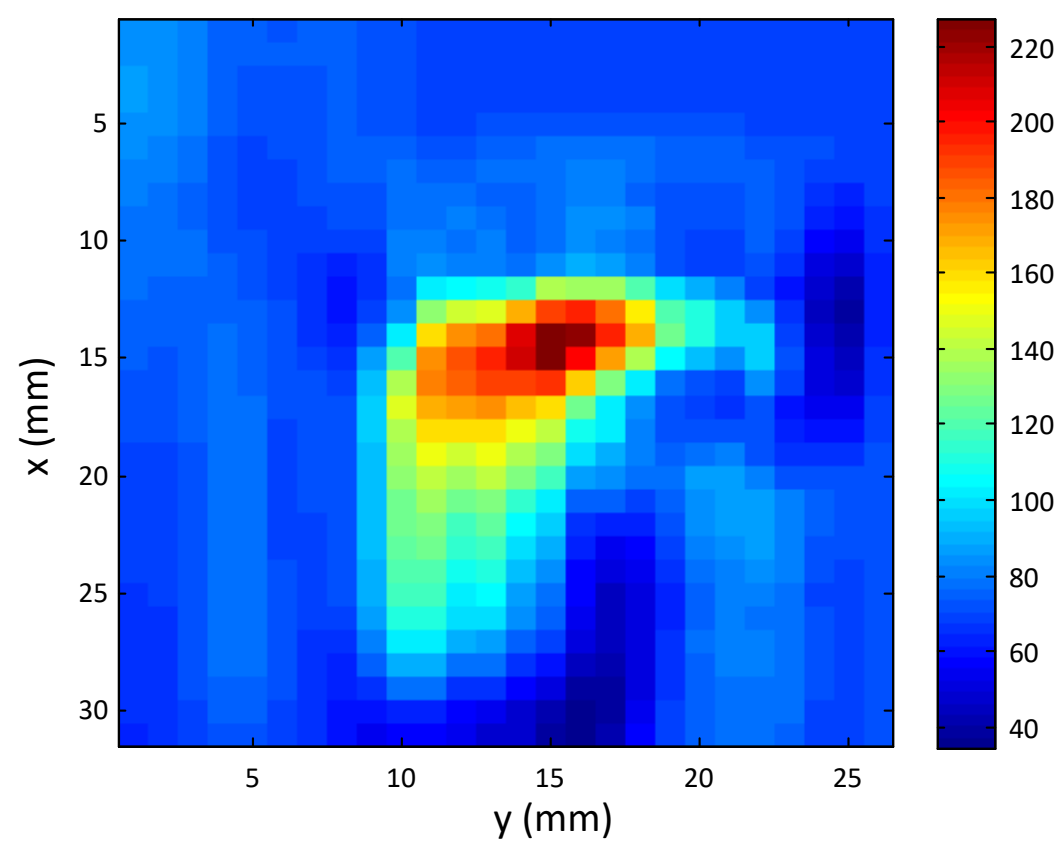

(b)

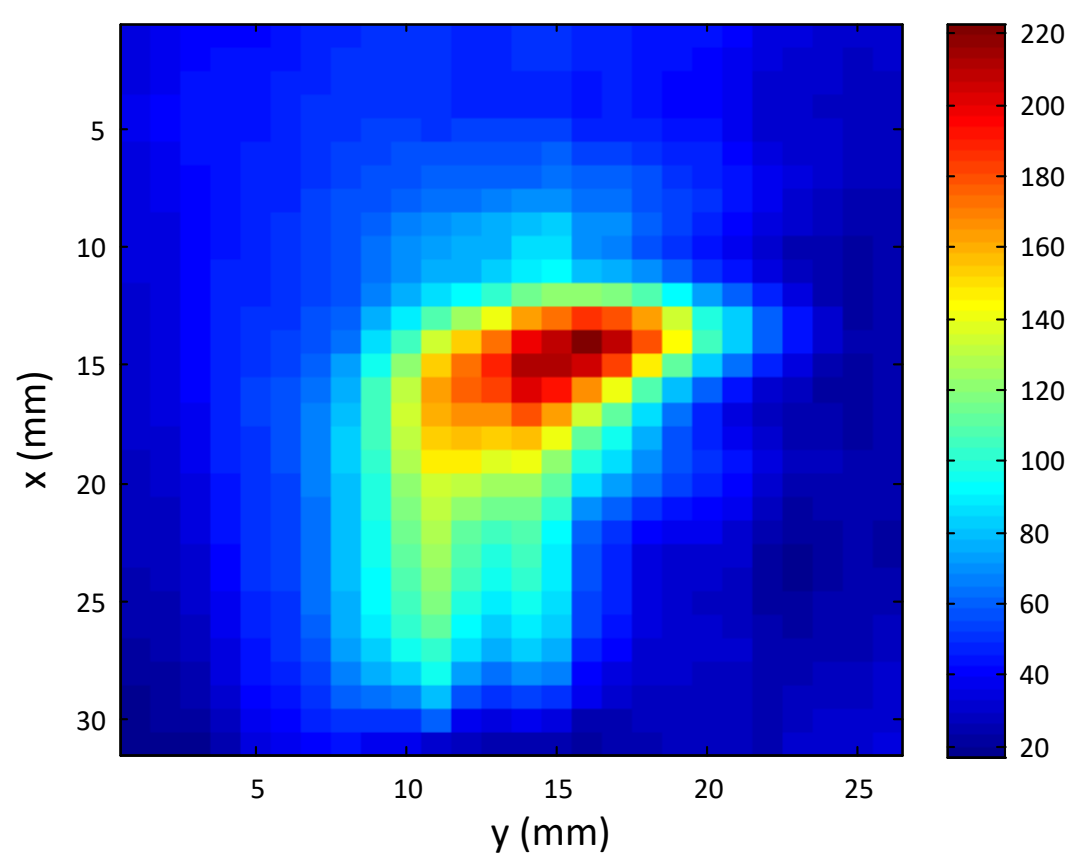

(c)

Figure 32. Measurements of the hidden L-shaped metal layer with CW stimulus at (a) $90 \mathrm{GHz}$, (b) $94 \mathrm{GHz}$ and (c) $97 \mathrm{GHz}$ (x and y coordinates in $\mathrm{mm}$ )

\section{B. 3 Detected voltage mapping with broadband noisy stimulus (illuminated passive system)}

Two cases were considered, depending on whether the BPF was inserted between the LNAs and the detector, or not. The sample was the one with the hidden different dielectric L-shaped. 


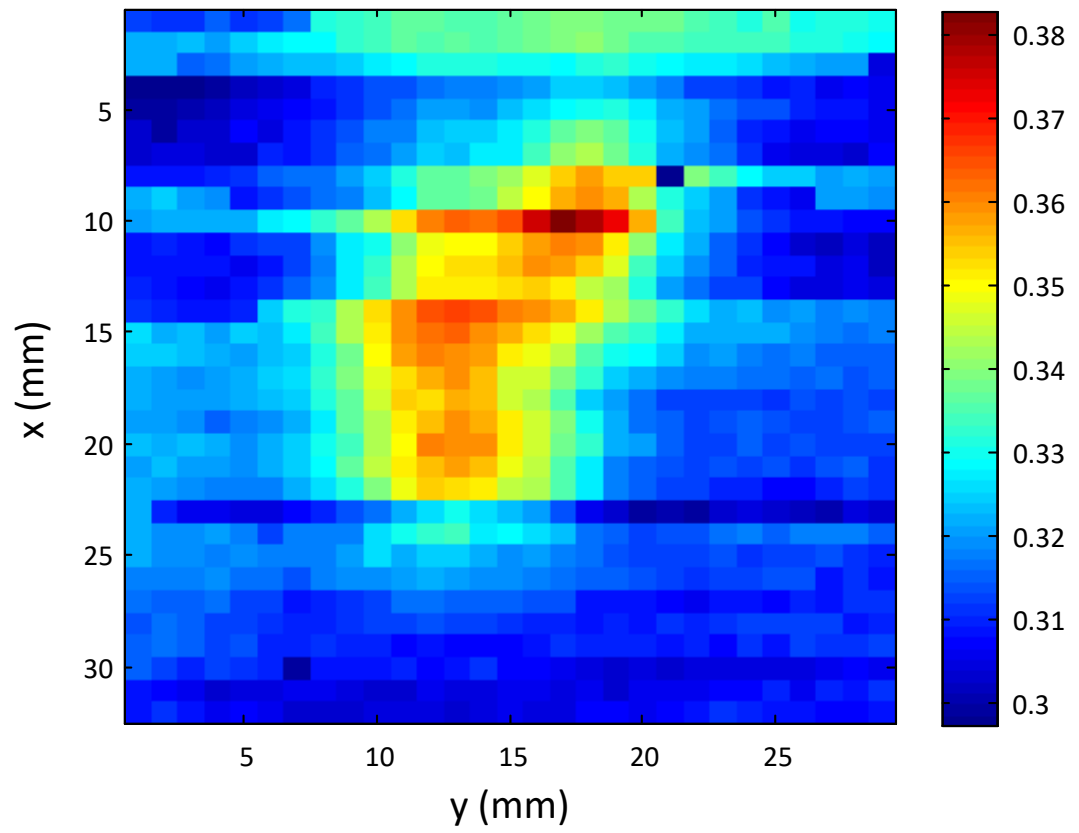

Figure 33. Measured image with illuminated passive system with no BPF filter included ( $\mathrm{x}$ and $\mathrm{y}$ coordinates in mm)

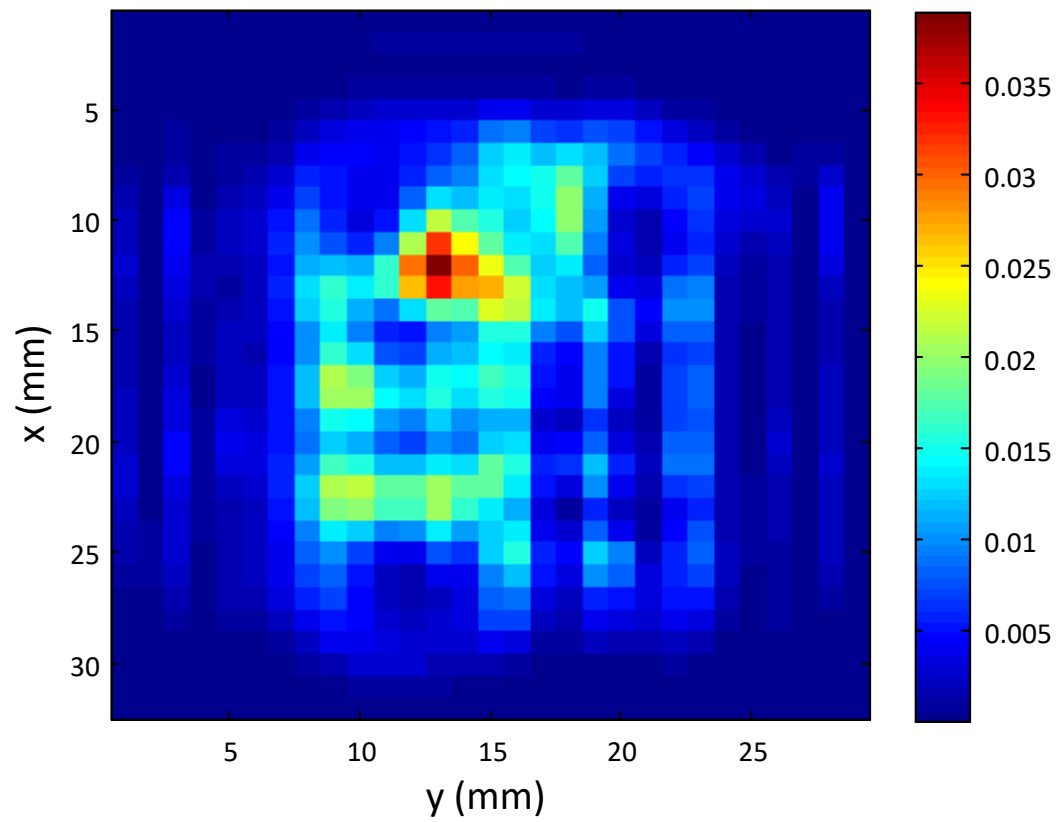

Figure 34. Simulated image with illuminated passive system with no BPF filter included ( $\mathrm{x}$ and $\mathrm{y}$ coordinates in mm) 


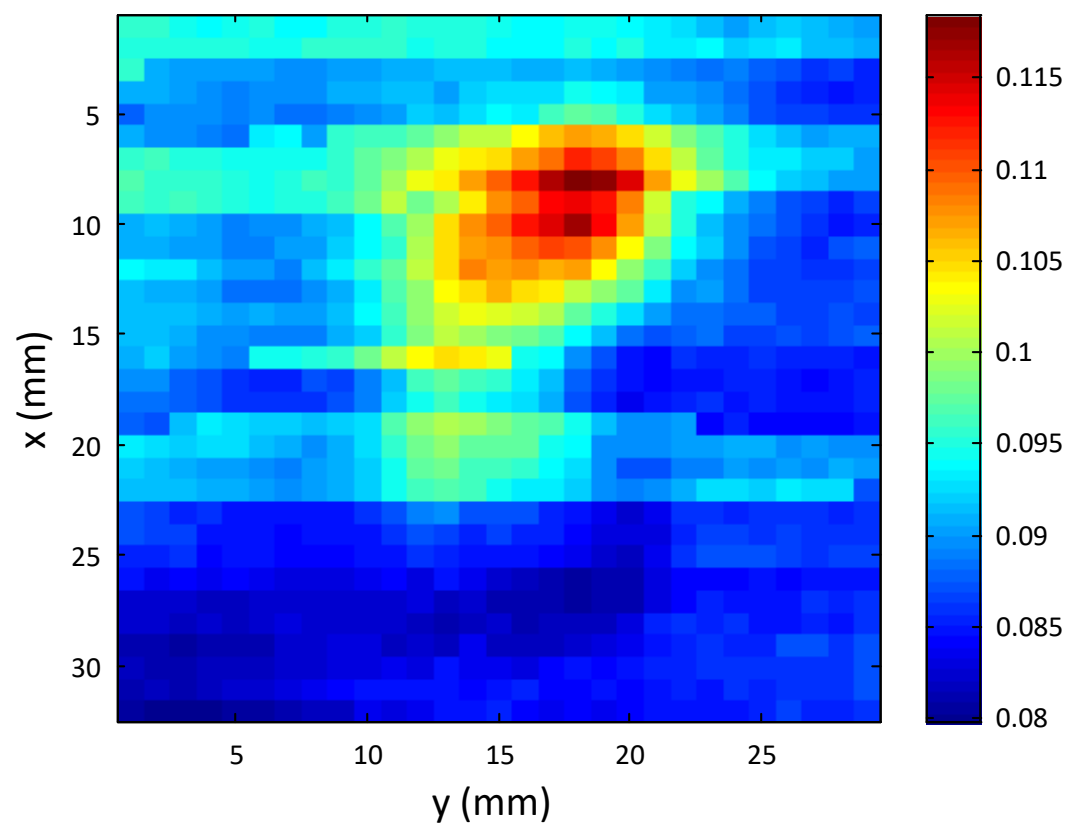

Figure 35. Measured image with illuminated passive system with BPF filter included (x and y coordinates in mm)

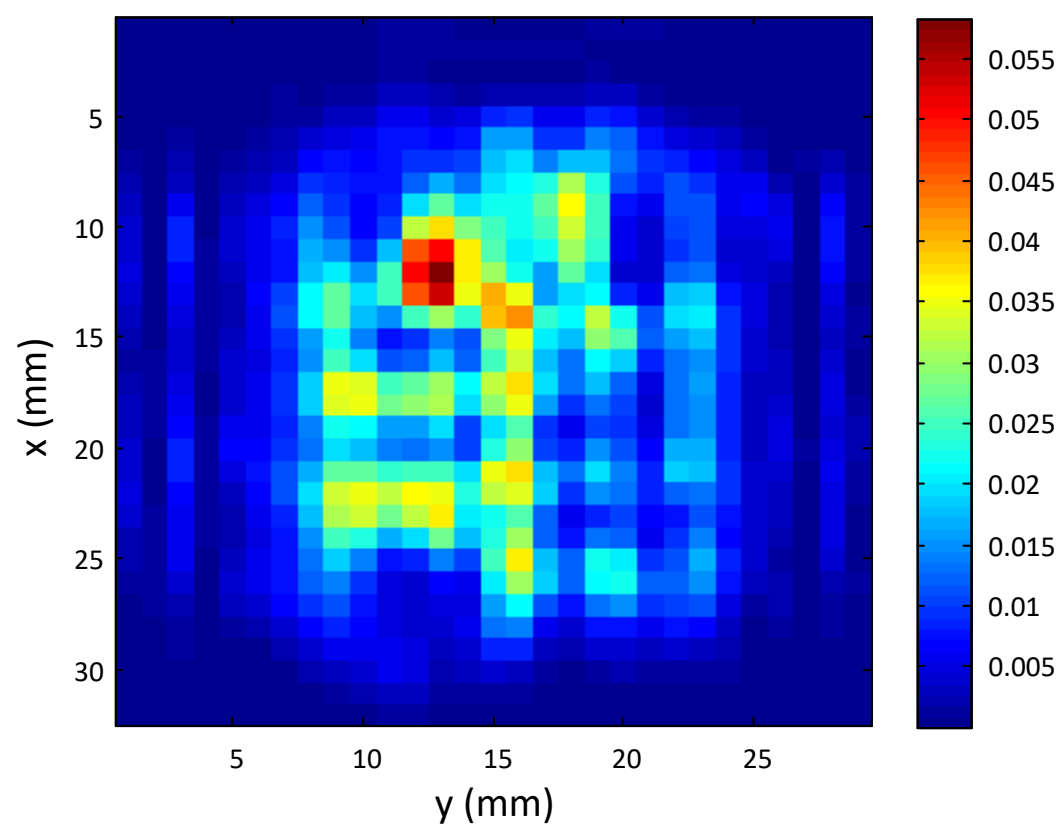

Figure 36. Simulated image with illuminated passive system with BPF filter included (x and y coordinates in mm)

While in simulations the presence of the filter has minimal effect (correlation with reference 0.5587 without filter, 0.5225 with filter), the measured image without filter resembles better the reference image (correlation with reference 0.617 without filter, 0.494 with filter). Nevertheless, the circulator is present in both setups limiting the frequency range, but less than the filter. Differences in measured and simulated output voltages correspond to differences in the range of RF gain and power levels around $3 \mathrm{~dB}$.

\section{CONCLUSIONS}

The interface between transceiving and radiating parts of basic imaging systems is discussed from the perspective of circuit and system performance simulation. A method was proposed to emulate the radiated emissivity profile of a target body in a passive scenario. A new procedure to use harmonic balance simulation to emulate non-linear circuit/system response to a broadband noisy stimulus was also 
proposed. A simulation procedure was described to link both aspects and it was tested with basic experiments. Of course, its reliability is based on the reliability of partial simulations, which unfortunately is limited, and still with room for improvement in the comparison with measurements, particularly for complex scenarios. Passive and active setups, CW and broadband in $\mathrm{W}$ band were considered. The passive setup in an indoor environment requires higher sensitivity or additional illumination, which converts the system in active. Higher broadband gain means that noise power levels are prone to saturation. A passive noisy broadband illuminated system seems the most feasible alternative.

The RF to dc system response may have a relevant impact on global system performance. In this case, $\mathrm{RF}$ to dc response shows remarkable peaks compared to the pure RF response, due to the addition of a detector and its sensitivity at higher frequencies. Interaction between the RF chain response and detector response produces a reduction in effective bandwidth and, at the same time, the dc response goes beyond the band defined by the RF parts (filter and circulator). A broad operation band may produce cancellation in the maximum and minimum detected intensities and the consideration of interaction between optical parts and the RF response may help to prevent them. The simulation procedure is suitable to be modified for more complex imaging systems, such as FMCW radars.

For the sake of clarity TABLE III summarizes figures listed indicating the conditions (measured or simulated, hidden L shape of metal or dielectric, stimulus frequency range, etc.) and the numerical correlation with the image of reference.

TABLE III. Correlation of the different images listed with the image of reference Figure 17 and corresponding conditions

\begin{tabular}{|c|c|c|c|c|}
\hline Figure & $\begin{array}{c}\text { Measurements/ } \\
\text { Simulation }\end{array}$ & Conditions & Target & $\begin{array}{c}\text { Correlation with } \\
\text { Reference }\end{array}$ \\
\hline 18 & Simulation & W band & Dielectric & 0.5215 \\
\hline 19 & Simulation & $97-110 \mathrm{GHz}$ & Dielectric & 0.6053 \\
\hline 20 & Measurements & Pure Passive & Dielectric & 0.2315 \\
\hline 21 & Measurements & $\mathrm{S}_{11}$ at $90 \mathrm{GHz}$ & Metal & 0.2732 \\
\hline 22 & Measurements & $\mathrm{S}_{11}$ at $94 \mathrm{GHz}$ & Metal & 0.4041 \\
\hline 23 & Measurements & $\mathrm{S}_{11}$ at $90 \mathrm{GHz}$ & Dielectric & 0.3306 \\
\hline 24 & Measurements & $\mathrm{S}_{11}$ at $94 \mathrm{GHz}$ & Dielectric & 0.2162 \\
\hline 25 & Measurements & {$\left[\mathrm{S}_{11}\right]^{2}$ in $90-100 \mathrm{GHz}$} & Dielectric & 0.3358 \\
\hline 26 & Measurements & {$\left[\mathrm{S}_{11}\right]^{2}$ in $75-100 \mathrm{GHz}$} & Dielectric & 0.3443 \\
\hline $32 \mathrm{a}$ & Measurements & $\mathrm{CW}$ at $90 \mathrm{GHz}$ & Metal & 0.3037 \\
\hline $32 b$ & Measurements & $\mathrm{CW}$ at $94 \mathrm{GHz}$ & Metal & 0.3829 \\
\hline $32 \mathrm{c}$ & Measurements & $\mathrm{CW}$ at $97 \mathrm{GHz}$ & Metal & 0.3389 \\
\hline 33 & Measurements & Illum. No BPF & Dielectric & 0.6170 \\
\hline 34 & Simulation & Illum. No BPF & Dielectric & 0.5587 \\
\hline 35 & Measurements & Illum. BPF & Dielectric & 0.4940 \\
\hline 36 & Simulation & Illum. BPF & Dielectric & 0.5225 \\
\hline
\end{tabular}




\section{REFERENCES}

1. J.A. Nanzer, Microwave and millimeter-wave remote sensing for security applications, Artech House, 2012, 372 pages

2. F.T. Ulaby, R.K. Moore, A.K. Fung, Microwave Remote Sensing: Active and Passive, Vol. I: Microwave Remote Sensing Fundamentals and Radiometry, Addison-Wesley, Advanced Book Program, Reading, Massachusetts, 1981, 456 pages

3. H. Essen, H.H. Fuchs, M. Hagelen, [et al], Concealed Weapon Detection with Active and Passive Millimeterwave Sensors, Two Approaches, German Microwave Conference, GeMiC 2006, Karlsruhe, Germany, March 28-30, 2006

4. K. Won-Gyum, M. Nam-Won, K. Hwang-Kyeom and K. Yong-Hoon, Linear Polarization Sum Imaging in Passive Millimeter-Wave Imaging System for Target Recognition. Progress In Electromagnetics Research, Vol. 136, 2013, pp. 175-193

5. K. Won-Gyum, M. Nam-Won, J.-M. Kang, and K. Yong-Hoon, Loss Measuring of Large Aperture Quasi-Optics for W-Band Imaging Radiometer System. Progress In Electromagnetics Research, Vol. 125, 2012, pp. 295-309

6. B. Gonzalez-Valdes, G. Allan, Y. Rodriguez-Vaqueiro, [et al], Sparse Array Optimization Using Simulated Annealing and Compressed Sensing for Near-Field Millimeter Wave Imaging, IEEE Transactions on Antennas and Propagation, Vol. 62, Issue 4, April 2014, pp. 1716 - 1722

7. T. Nozokido, Millimeter-Wave Active and Passive Microscopies, International Symposium on Electromagnetic Theory (EMTS), 2013, pp. 25-28

8. K.B. Cooper, R.J. Dengler, N. Llombart, B. Thomas, G. Chattopadhyay, P.H. Siegel, THz Imaging Radar for Standoff Personnel Screening, IEEE Transactions on Terahertz Science and Technology Vol. 1, Issue 1, Sept. 2011, Page(s):169 - 182

9. J.P. Pascual, B. Aja, M.L. de la Fuente, [et al], System simulation of a differential radiometer using standard RF microwave simulators, SIMULATION: Transactions of the Society for Modeling and Simulation International, Vol. 81, Issue 11, Nov. 2005, pp. 735-755

10. Y. Jiao, Y. Zhang, Y. Fan, Research on Modeling of W-band Wideband Total Power Radiometer Channel, Journal of Electromagnetic Waves and Applications, Vol. 23, Issue 8-9, 2009, pp. 10491058

11. Application Note: OCW Coffee-Can Radar Optimized in NI AWR Software. http://www.awrcorp.com/sites/default/files/resource-library/attachments/AN-V-RDR-CFCN2017.5.5.pdf

12. D.M. Sheen, D.L. Mcmakin, T.E. Hall, Three-Dimensional Millimeter-Wave Imaging for Concealed Weapon Detection. IEEE Transactions on Microwave Theory and Techniques, , Vol. 49, No. 9, Sept. 2001, pp. 1581-1592

13. O. Yurduseven, Indirect Microwave Holographic Imaging of Concealed Ordnance for Airport Security Imaging Systems, Progress In Electromagnetics Research, Vol. 146, 2014 pp. 7-13

14. E. Artal, B. Aja, M.L. de la Fuente, J.P. Pascual, A. Mediavilla, [et al], LFI 30 and $44 \mathrm{GHz}$ receivers Back-End Modules, Journal of Instrumentation, Vol. 4, Dec. 2009

15. http://www.keysight.com/en/pc-1297113/advanced-design-system-ads?cc=US\&lc=eng

16. F.J. Casas, J.P. Pascual, M.L. de la Fuente, E. Artal, J. Portilla, Simple nonlinearity evaluation and modeling of low-noise amplifiers with application to radio astronomy receivers, Review of Scientific Instruments, July 2010; 81(7):074704. DOI: 10.1063/1.3463295

17. H. Togo, T. Kojima, S. Mochizuki, [et al], Reconstruction of MMW Near-field Image with Antenna-radiation-pattern Deconvolution Processing, Proceedings of 2013 URSI International Symposium on Electromagnetic Theory (EMTS). 20-24 May 2013. Hiroshima, Japan

18. J.V. Terán, D. Vegas, E. Artal, Filtro en banda W con alto rechazo en banda imagen, URSI 2015, XXX Simposium Nacional de la Unión Científica Internacional de Radio, Pamplona, 2-4 Sept. 2015

19. J.L. Cano, E. Villa, V. Terán [et al], A W-band polarimeter for radio astronomy applications: Design and simulation”. International Conference on Electromagnetics in Advanced Applications (ICEAA), Torino, Italy, 7-11 Sept. 2015 
20. https://www.mathworks.com/products/matlab.html

21. S. Reyaz, A. Gustafsson, C. Samuelsson, R. Malmqvist, [et al], A W-band RF-MEMS switched LNA in a $70 \mathrm{~nm}$ mHEMT process. International Journal of RF and Microwave Computer-Aided Engineering, Vol. 25, Issue 7, Sept. 2015, pp. 639-646. DOI:10.1002/mmce.20902

\section{ACKNOWLEDGMENT}

The authors would like to thank the University of Cantabria Industrial Doctorate programme 2014, project: "Estudio y Desarrollo de Tecnologías para Sistemas de Telecomunicación a Frecuencias Milimétricas y de Terahercios con Aplicación a Sistemas de Imaging en la Banda 90 GHz-100GHz” and the Spanish Ministry of Economy, Science and Innovation for the financial support provided through projects CONSOLIDER-INGENIO CSD2008-00068 (TERASENSE) and the continuing excellence network SPATEK and the project TEC2014-58341-C4-1-R and TEC2017-83343-C4-1-R.

The authors would like to express their gratitude to all the staff of DICOM's Microwaves \& RF group for their help and to the assembling laboratory staff: Ana Pérez, Eva Cuerno and Sandra Pana for their help with the fabrication of the prototypes and to Dermot Erskine for the revision of the text. 